\title{
Directional Redundancy for Robot Control
}

\author{
Nicolas Mansard and François Chaumette, Member, IEEE
}

\begin{abstract}
The paper presents a new approach to design a control law that realizes a main task with a robotic system and simultaneously takes supplementary constraints into account. Classically, this is done by using the redundancy formalism. If the main task does not constrain all the motions of the robot, a secondary task can be achieved by using only the remaining degrees of freedom (DOF). We propose a new general method that frees up some of the DOF constrained by the main task in addition of the remaining DOF. The general idea is to enable the motions produced by the secondary control law that help the main task to be completed faster. The main advantage is to enhance the performance of the secondary task by enlarging the number of available DOF. In a formal framework, a projection operator is built which ensures that the secondary control law does not delay the completion of the main task. A control law can then be easily computed from the two tasks considered. Experiments that implement and validate this approach are presented. The visual servoing framework is used to position a six-DOF robot while simultaneously avoiding occlusions and joint limits.
\end{abstract}

Index Terms-Gradient projection method, redundancy, robot control, sensor-feedback control, visual servoing.

\section{INTRODUCTION}

C LASSICAL control laws in robotics are based on the minimization of a task function which corresponds to the realization of a given objective. Usually, this main task only concerns the position of the robot with respect to a target and does not take into account the environment where the robot evolves. However, to integrate the servo into a real robotic system, the control law should also make sure that it takes into account any necessary constraints such as avoiding undesirable configurations (joint limits, kinematic singularities, or sensor occlusions).

Two very different approaches have been proposed in the literature to deal with this problem. A first solution is to take into account the whole environment from the very beginning into a single minimization process, for example by pre-computing a trajectory to be followed to the desired position [18] or by designing a specific global navigation function [6]. This provides a complete solution, which ensures the obstacles avoidance when moving to complete the main task. However, the pre-computations (construction of the trajectory or of the navigation function) require a lot of knowledge about the environment, and in

Manuscript received December 21, 2005; revised February 20, 2007. First published May 27, 2009; current version published June 10, 2009. This paper was presented in part at the IEEE 44th IEEE Conference on Decision and Control and European Control Conference (ECC-CDC'05), Sevilla, Spain, December, 2005. Recommended by Associate Editor J. P. Hespanha.

N. Mansard was with INRIA Rennes-Bretagne Atlantique-IRISA, Rennes 35042, France and is now with LAAS, CNRS, Toulouse 31077, France (e-mail: nicolas.mansard@laas.fr).

F. Chaumette is with INRIA Rennes-Bretagne Atlantique-IRISA, Rennes 35042, France (e-mail: francois.chaumette@irisa.fr).

Color versions of one or more of the figures in this paper are available online at http://ieeexplore.ieee.org.

Digital Object Identifier 10.1109/TAC.2009.2019790 particular about the constraints to take into account. This solution is thus less reactive to changes in the goal, in the environment or in the constraints.

Rather than taking into account the whole environment from the very beginning, another approach considers the secondary constraints through an objective function to be locally minimized. This provides very reactive solutions, since it is very easy to modify the objective function during the servo. A first solution to take the secondary objective function into account is to realize a trade off between the main task and the constraints [19]. In this approach, the control law generates motions that try to make the main task function decrease and simultaneously take the robot away from the kinematic singularities and the joint limits. On the opposite, a second solution is to dampen any motion that does not respect the constraints. This solution has been applied using the weighted least norm solution to avoid joint limits [3]. The control law does not induce any motion to take the robot away from the obstacles, but it forbids any motion in their direction. Thus, it avoids oscillations and unnecessary motions.

However, these two methods can strongly disturb the execution of the main task. A second specification is thus generally added: the secondary constraints should not disturb the main task. The Gradient Projection Method (GPM) has been initially introduced for non-linear optimization [20] and applied then in robotics [7], [10], [15], [21]. The constraints are embedded into a cost function [13]. The gradient of this cost function is computed as a secondary task that moves the robot aside the obstacles. This gradient is then projected onto the set of motions that keep the main task invariant and added to the first part of the control law that performs the main task. The main advantage of this method with respect to [19] and [3] is that, thanks to the choice of the adequate projection operator, the secondary constraints have no effect on the main task. The redundancy formalism has been used in numerous works to manage secondary constraints, for example force distribution for the legs of a walking machine [14], occlusion and joint-limit avoidance [17], multiple hierarchical motions of virtual humanoids [1], or human-motion filtering under constraints imposed by the patient anatomy for human-machine cooperation using vision-based control [9]. However, since the secondary task is performed under the constraint that the main task is realized, the avoidance contribution can be so disturbed that it becomes inefficient. In fact, only the degrees of freedom (DOF) not controlled by the main task can be exploited to perform the avoidance. The more complicate the main task is, the more DOF it uses, and the more difficult to apply the secondary constraints. Of course, if the main task uses all the DOF of the robot, no secondary constraint can be taken into account.

Nevertheless, even if a DOF is controlled by the primary control law, the constraints should be taken into account if it "goes 
in the same way" as the main task. Imposing the control law part due to the constraints to let the main task invariant can be a too strong condition. We rather propose in this article a more general solution that only imposes to the secondary control law not to increase the error of the main task (to preserve the stability of the system), that is when the secondary task goes in the same direction than the main task. This method is thus called directional redundancy. By this way, the free space on which the gradient is projected is larger. More DOF are thus available for any secondary constraint, and the control law derived from the applied constraints is less disturbed.

The paper is organized as follow. In Section II, we recall the classical redundancy formalism and build using the same continuous approach a new projection operator that enlarges the projection domain. We then propose in Section III to use a sampled approach to compute a similar projection operator, in order to improve the global behavior of the system. If the control law due to the main task is stable, which is the usual hypothesis, the obtained control law is proved to be stable, and to asymptotically converge to the completion of the main task and to the best local minimum of the secondary task (Section IV). For the experiments, the proposed method has been applied to a visual servoing problem. Visual servoing consists in a closed loop reacting to image data [4], [7], [8], [11], [12]. It is a typical problem where the constraints of the workspace are not considered into the main task. The visual servoing framework is quickly presented in Section V. Finally, Section VI describes several experiments that show the advantages of the proposed method.

\section{DiRECTIONAL REDUNDANCY USING A CONTINUOUS APPROACH}

In this section, the classical redundancy formalism is first recalled. Based on this formulation, we deduce a more general way to take the secondary term into account, by enlarging the free space of the main task. The stability of this new control law is then proved. We conclude by explaining the limitations of this control scheme. To solve these limitations, a second-order derivation is necessary, as done in Section III.

\section{A. Considering Only the Main Task}

Let $\mathbf{q}$ be the joint position of the robot. The main task function is e. It is based on features extracted from the sensor output. The robot is controlled using the joint velocities $\dot{\mathbf{q}}$. The Jacobian of the main task $\mathbf{e}$ is $\mathbf{J}$ defined by

$$
\dot{\mathbf{e}}=\frac{\partial \mathbf{e}}{\partial \mathbf{q}} \dot{\mathbf{q}}=\mathbf{J} \dot{\mathbf{q}} \text {. }
$$

Let $n$ be the number of DOF of the robot $(n=\operatorname{dim} \mathbf{q})$ and $m$ be the size of the main task $(m=\operatorname{dim} \mathbf{e} \leq n)$. The task function is designed to be full rank, i.e. $\operatorname{rank}(\mathbf{J})=m[21]$.

The task function $\mathrm{e}$ is the controller input, and the robot joint velocity $\dot{\mathbf{q}}$ is the controller output. The controller has to regulate the input to $\mathbf{0}$ according to a decreasing behavior $\dot{\mathbf{e}}^{*}$ chosen when designing the control law. By inverting (1), the joint motion $\dot{\mathbf{q}}$ that realizes this required decrease $\dot{\mathbf{e}}^{*}$ of the error is given by the least-square inverse

$$
\dot{\mathbf{q}}=\mathbf{J}^{+} \dot{\mathbf{e}}^{*}
$$

where the notation $\mathbf{A}^{+}$refers to the Moore-Penrose inverse of the matrix A [2]. By (2), we consider that the Jacobian matrix is perfectly known. If it is not the case, due to inaccuracy in the calibration process or uncertainties in the robot-scene model, an approximation $\widehat{\mathbf{J}^{+}}$has to be used instead of $\mathbf{J}^{+}$. It is possible to prove that (2) is stable if $f \mathbf{J} \widehat{\mathbf{J}+}>0$ [21]. In the following, we make the assumption that $\mathbf{J}$ is perfectly known, from which we deduce $\mathbf{J J}^{+}=\mathbf{I}_{\mathbf{m}}$. We also assume that $\dot{\mathbf{e}}^{*}$ is a stable vector field, that is to say it is possible to find a correct Lyapunov function $V(\mathbf{e})$ so that if $\dot{\mathbf{e}}=\dot{\mathbf{e}}^{*}$, then $\dot{V}<0$ and (2) is asymptotically stable.

It is classical to specify the control scheme by an exponential decoupled decreasing of the error function, by imposing $\dot{\mathrm{e}}^{*}=-\lambda \mathbf{e}$, where $\lambda$ is a positive parameter that tunes the convergence speed. This finally produces the classical control law $\dot{\mathbf{q}}=-\lambda \mathbf{J}+\mathbf{e}$. In that case, a classical Lyapunov function is based on the norm $\|\mathbf{e}\|$, e.g. $V(\mathbf{e})=(1 / 2)\|\mathbf{e}\|^{2}$.

\section{B. Classical Redundancy Formalism}

The solution (2) computed above is only one particular solution of (1): it is the solution of least norm that realizes the reference motion $\dot{\mathrm{e}}^{*}$

$$
\dot{\mathbf{q}}=\min \left\{\mathbf{z} \backslash \mathbf{J z}=\dot{\mathbf{e}}^{*}\right\}
$$

The redundancy formalism [21] uses a more general solution which enables to consider a secondary criterion. The robot motion is given by

$$
\dot{\mathbf{q}}=\mathbf{J}^{+} \dot{\mathbf{e}}^{*}+\mathbf{P z}
$$

where $\mathbf{P}$ is the projection operator onto the null space of the matrix $\mathbf{J}$ (i.e., $\mathbf{P}=\mathbf{I}_{\mathbf{n}}-\mathbf{J}^{+} \mathbf{J}$ ), and $\mathbf{z}$ is an arbitrary vector, used to apply a secondary control law. Thanks to $\mathbf{P}$, this secondary motion $\mathbf{z}$ is performed without disturbing the main task e having priority.

It is easy to check that the joint motion $\dot{\mathbf{q}}$ given by (4) produces exactly the specified motion $\dot{\mathbf{e}}^{*}$ in the task function space

$$
\dot{\mathrm{e}}=\mathbf{J} \dot{\mathbf{q}}=\mathbf{J} \mathbf{J}^{+} \dot{\mathrm{e}}^{*}+\mathbf{J P z}=\dot{\mathrm{e}}^{*}+\mathbf{0}
$$

since $\mathbf{J J}^{+}=\mathbf{I}_{\mathbf{m}}$ and $\mathbf{J P}=\mathbf{J}\left(\mathbf{I}_{\mathbf{n}}-\mathbf{J}^{+} \mathbf{J}\right)=\mathbf{0}$. The joint motion $\dot{\mathbf{q}}$ is chosen to realize exactly the motion $\dot{\mathbf{e}}^{*}$ in the task function space, and to perform at best a secondary task whose corresponding joint motion is $\mathbf{z}$. Looking at (3), the motion $\dot{\mathbf{q}}$ obtained in (4) is simply another solution of (1), however not minimal in norm.

It is easy to check that the addition of the secondary term does not modify the stability of the control law. Let $V(\mathbf{e})$ be a Lyapunov function associated to control law (2) (that is to say such that $\dot{V}=(\partial V / \partial \mathbf{e}) \dot{\mathbf{e}}<0)$. Then $V$ is also a correct 
Lyapunov function for the control law (4). In fact, $\dot{V}$ does not depend of the secondary term $\mathbf{z}$

$$
\dot{V}=\frac{\partial V}{\partial \mathbf{e}} \mathbf{J} \dot{\mathbf{q}}=\frac{\partial V}{\partial \mathbf{e}}\left(\mathbf{J} \mathbf{J}^{+} \dot{\mathbf{e}}^{*}+\mathbf{J P z}\right)=\frac{\partial V}{\partial \mathbf{e}} \dot{\mathbf{e}}^{*}=\dot{V}^{*}
$$

where $\dot{V}^{*} \triangleq(\partial V / \partial \mathbf{e}) \dot{\mathbf{e}}^{*}<0$ is the reference decrease speed, obtained when considering only the main task.

\section{Extension of the Convergence Condition}

In the classical redundancy formalism, the secondary control law $\mathbf{z}$ is applied under the condition that it does not modify the convergence speed $\dot{V}$, that is to say under the condition $\dot{V}=$ $\dot{V}^{*}$. However, to guarantee the convergence of the main task, it is sufficient to ensure that the convergence is at least as fast as $\dot{V}^{*}$. The condition of application of the secondary control law $\mathbf{z}$ can thus be written

$$
\dot{V} \leq \dot{V}^{*}
$$

In the following, we propose a solution to apply the secondary control law $\mathbf{z}$ under Condition (7). By analogy with the classical redundancy formalism, we search a control law of the following form:

$$
\dot{\mathbf{q}}=\mathbf{J}^{+} \dot{\mathbf{e}}^{*}+\dot{\mathbf{q}}_{2} .
$$

We search the condition on $\dot{\mathbf{q}}_{2}$ such that this control law respects (7). When (8) is applied, $\dot{V}$ can be written

$$
\dot{V}=\frac{\partial V}{\partial \mathbf{e}} \mathbf{J} \dot{\mathbf{q}}=\frac{\partial V}{\partial \mathbf{e}}\left(\dot{\mathbf{e}}^{*}+\mathbf{J} \dot{\mathbf{q}}_{2}\right)=\dot{V}^{*}+\frac{\partial V}{\partial \mathbf{e}} \mathbf{J} \dot{\mathbf{q}}_{2}
$$

By introducing this last equation in (7), a simpler form of the condition is obtained

$$
\nabla V^{\top} \mathbf{J} \dot{\mathbf{q}}_{2} \leq 0
$$

where $\nabla V=(\partial V / \partial \mathbf{e})^{\top}$.

\section{Construction of the Extended Projection}

In this section we build an operator that projects any secondary control law to keep only the part respecting (10). Let $\mathbf{z}$ be any secondary control law. We note $\dot{\mathbf{q}}_{2}=\mathbf{P z}$, and we search $\mathbf{P}$ so that $\dot{\mathbf{q}}_{2}$ respects (10).

To simplify the formulation, adequate bases of both joint and task function spaces are chosen. Let $\mathbf{U}, \boldsymbol{\Sigma}$ and $\mathbf{V}$ be the result of the SVD of $\mathbf{J}$ :

$$
\mathbf{J}=\mathbf{U} \boldsymbol{\Sigma} \mathbf{V}^{\top}
$$

with $\mathbf{V}$ a basis of the joint space, $\mathbf{U}$ a basis of the task function space, $\boldsymbol{\Sigma}=\left[\Delta_{\sigma} \mathbf{0}\right]$, and $\Delta_{\sigma}$ the diagonal matrix whose coefficients are the $m$ singular values of $\mathbf{J}$, noted $\sigma_{i}\left(\sigma_{i}>0\right)$. Condition (10) can thus be written

$$
\nabla V^{\top} \mathbf{U} \boldsymbol{\Sigma} \mathbf{V}^{\top} \dot{\mathbf{q}}_{2}=\widetilde{\nabla V}^{\top} \boldsymbol{\Sigma} \widetilde{\dot{\mathbf{q}}}_{2} \leq 0
$$

where $\widetilde{\nabla V}=\mathbf{U}^{\top} \nabla V$ and $\widetilde{\mathbf{q}}_{2}=\mathbf{V}^{\top} \dot{\mathbf{q}}_{2}$. To simplify the construction of the projection operator, this condition is restricted to the following:

$$
\forall i \in[1 \ldots m], \quad \widetilde{v_{i}} \sigma_{i} \widetilde{\dot{q}_{2_{i}}} \leq 0
$$

where $\widetilde{\nabla V}=\left(\widetilde{v_{1}}, \ldots, \widetilde{v_{m}}\right)$. This condition is more restrictive than the previous one. However, it ensures a better behavior of the robot. Particularly, if the Lyapunov function is the norm of the error (as classically done), this condition ensures the convergence of each singular components of the error separately, while (10) ensures only the convergence of the norm of the error, which can lead to a temporary increase of one or several components. This can cause some troubles during the servo. For example, in visual servoing, it can cause the loss of some features during the robot motion. By (13), we ensure that each component will be faster than the required motion $\dot{\mathrm{e}}^{*}$, avoiding thus such problems.

Let us now consider any secondary control law $\mathbf{z}$. We note $\widetilde{\mathbf{z}}=\mathbf{V z}$. To build a secondary term $\dot{\mathbf{q}}_{2}$ from $\mathbf{z}$ that respects (13), we just have to keep the components that respects the inequality, and to nullify the others: for all $i \in[1 \ldots m]$

$$
\widetilde{q_{z_{i}}}= \begin{cases}\widetilde{z_{i}} & \text { if } i>m \text { or } z_{i}=0 \\ \widetilde{z_{i}} & \text { if } \widetilde{z_{i}} \text { and }\left(\widetilde{v_{i}} \sigma_{i}\right) \text { have opposite signs . } \\ 0 & \text { if } \widetilde{z_{i}} \text { and }\left(\widetilde{v_{i}} \sigma_{i}\right) \text { have the same sign }\end{cases}
$$

This equation can be put under a matricial form

$$
\widetilde{\dot{\mathbf{q}}} 2=\widetilde{\mathbf{P}}(\widetilde{\mathbf{z}})=\widetilde{\mathbf{P}_{\mathbf{z}}} \widetilde{\mathbf{z}}=\left(\begin{array}{ccc}
p_{1}(\widetilde{\mathbf{z}}) & & 0 \\
& \ddots & \\
0 & & p_{n}(\widetilde{\mathbf{z}})
\end{array}\right) \widetilde{\mathbf{z}}
$$

where the components $p_{i}$ of $\widetilde{\mathbf{P}_{\widetilde{\mathbf{z}}}}$ are defined by

$$
p_{i}(\mathbf{z})= \begin{cases}1 & \text { if } i>m \text { or } \widetilde{z_{i}}=0 \\ 1 & \text { if } \widetilde{z_{i}} \text { and }\left(\widetilde{v_{i}} \sigma_{i}\right) \text { have opposite signs } . \\ 0 & \text { if } \widetilde{z_{i}} \text { and }\left(\widetilde{v_{i}} \sigma_{i}\right) \text { have the same sign }\end{cases}
$$

It has to be noticed that $\mathbf{P}$ is not linear: the associated matrix $\widetilde{\mathbf{P}_{\widetilde{z}}}$ is computed from $\mathbf{z}$. The term $\widetilde{\mathbf{P}_{\mathbf{z}} \mathbf{z}}$ is thus not linear in $\mathbf{z}$.

The control law that realizes the main task $\mathbf{e}$ and ensures that the secondary control law $\mathbf{z}$ respects Condition (13) can finally be written

$$
\dot{\mathbf{q}}=\mathbf{J}^{+} \dot{\mathbf{e}}^{*}+\mathbf{P}_{\widetilde{\mathbf{z}}} \mathbf{z}
$$

where $\mathbf{P}_{\mathbf{z}}=\mathbf{V} \widetilde{\mathbf{P}_{\widetilde{z}}} \mathbf{V}^{\top}$.

\section{E. Stability of the Control Law}

The computations that bring to the control law (16) prove the following result:

Theorem 2.1: Let $\mathbf{e}$ be any task function whose Jacobian $\mathbf{J}=$ $\partial \mathbf{e} / \partial \mathbf{q}$ is full rank. If the following control law:

$$
\dot{\mathbf{q}}=\mathbf{J}^{+} \dot{\mathbf{e}}^{*}+\mathbf{P}_{\mathbf{z}} \mathbf{z}
$$

is applied to the robotic system, where:

- $\dot{\mathrm{e}}^{*}$ is a stable vector field, that is to say it is possible to find an associated Lyapunov function $V(\mathbf{e})$ such that if $\dot{\mathbf{e}}=\dot{\mathbf{e}}^{*}$, then $\dot{V}<0$;

- $\mathbf{z}$ is any secondary control vector; then the error $\mathbf{e}$ asymptotically converges to zero.

\section{F. Potential Oscillations at Task Regulation}

The control law (16) is composed of two terms. The first one is linearly linked to $\dot{\mathbf{e}}^{*}$. The second term is $\dot{\mathbf{q}}_{2}=\mathbf{P}_{\mathbf{z}} \mathbf{z}$, whose only constraint is that each component has the proper sign. It is 


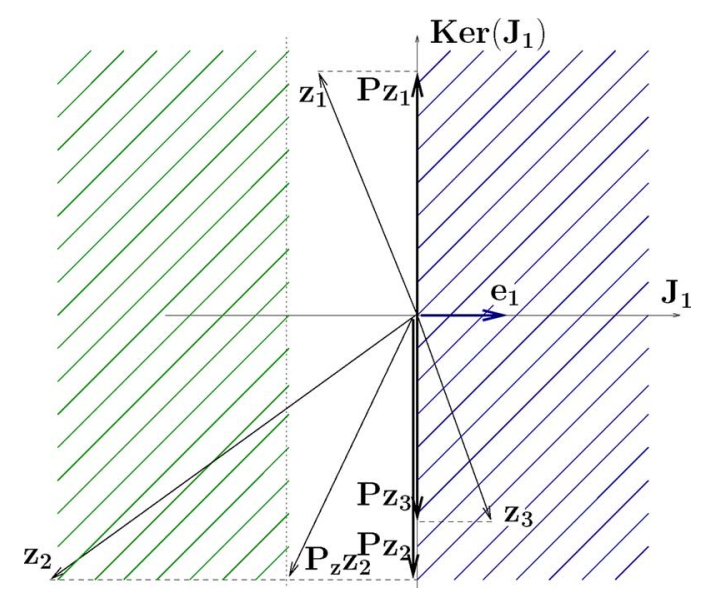

Fig. 1. Comparison of the free spaces using the classical redundancy framework, one bound (Section II) and two bounds (Section III). If the classical redundancy formalism is used, the secondary control law is projected onto the null space of the main task: the secondary terms $\mathbf{z}_{i}$ are projected on the corresponding $\mathbf{P} \mathbf{z}_{i}(i=1 \ldots 3)$. When only one bound is used, the free space is a "half-plane": the blue region is forbidden. The secondary terms $\mathbf{z}_{1}$ and $\mathbf{z}_{2}$ are not modified by the projection. However, the action of $\mathbf{z}_{2}$ on the main task is very important, and may result in oscillations or even instability, in particular if the time interval between two iterations is large. A second bound is then added: the green region is forbidden too. The secondary term $\mathbf{z}_{2}$ is then projected on $\mathbf{P}_{\mathbf{z}_{2}} \mathbf{z}_{2}$, which prevents any oscillation at task regulation.

thus not proportional to the error to regulate, and can be arbitrarily large when the main task converges to 0 .

When applied in practice, this control law may introduce oscillations when the main task converges, due to a too large value of $\dot{\mathbf{q}}_{2}$. The effect is very similar to the oscillations induced by a too large value of the gain on a sampled system. To correct this problem, it is necessary to introduce an upper bound on the value of the secondary term, in order to narrow the free space to a band whose width depends on the value of the main-task error (see Fig. 1). This upper bound appears directly when condition of projection is computed from a second-order expansion of the Lyapunov function, as it will be shown in the next section.

\section{DiRECTIONAL REDUNDANCY USING A DISCRETE APPROACH}

As explained in the previous paragraph, using only one bound can lead to oscillations when considering a sampled system. In this section, we present a solution to introduce a second bound. This requires to consider the robot as a discrete system.

As in the previous section, we want to ensure the convergence of the Lyapunov function at least as fast as when the main task is considered alone. The condition of application of the secondary control vector can thus be written

$$
V(\mathbf{e}+\Delta \mathbf{e}) \leq V\left(\mathbf{e}+\Delta \mathbf{e}^{*}\right)
$$

where $\Delta \mathbf{e}(t)=\mathbf{e}(t+\Delta t)-\mathbf{e}(t)$ and $\Delta \mathbf{e}^{*}(t)=\dot{\mathbf{e}}^{*}(t) \Delta t$ is the desired behavior of the main task and $\Delta t$ is the sampling interval.

In the previous section, we have considered a first order approximation of $V$. Let us now consider the second-order Taylor expansion of the Lyapunov function when the small displacement $\Delta \mathbf{e}$ is applied

$V(\mathbf{e}+\boldsymbol{\Delta} \mathbf{e})=V(\mathbf{e})+\frac{\partial V}{\partial \mathbf{e}} \boldsymbol{\Delta} \mathbf{e}+\frac{1}{2} \Delta \mathbf{e}^{\top} \frac{\partial^{2} V}{\partial \mathbf{e}^{2}} \boldsymbol{\Delta} \mathbf{e}+\mathcal{O}\left(\|\boldsymbol{\Delta} \mathbf{e}\|^{2}\right)$

where $\mathcal{O}\left(\|\Delta \mathbf{e}\|^{2}\right)$ denotes a term whose norm is asymptotically bounded by $\|\Delta \mathbf{e}\|^{2}$. Since $\Delta \mathbf{e}=\mathbf{J} \Delta \mathbf{q}+\mathcal{O}\left(\|\Delta \mathbf{q}\|^{2}\right)$ and since $\|\Delta q\|$ and $\|\Delta e\|$ are asymptotically of the same order, this last equation can be written

$$
\begin{array}{r}
V(\mathbf{e}+\boldsymbol{\Delta} \mathbf{e})=V(\mathbf{e})+\frac{\partial V}{\partial \mathbf{e}} \mathbf{J} \boldsymbol{\Delta} \mathbf{q}+\frac{1}{2} \Delta \mathbf{q}^{\top} \mathbf{J}^{\top} \frac{\partial^{2} V}{\partial \mathbf{e}^{2}} \mathbf{J} \Delta \mathbf{q} \\
+\mathcal{O}\left(\|\boldsymbol{\Delta} \mathbf{e}\|^{2}\right) .
\end{array}
$$

As previously, the control law can be searched under the following form, without any loss of generality:

$$
\Delta \mathbf{q}=\mathbf{J}^{+} \Delta \mathbf{e}^{*}+\Delta \mathbf{q}_{2}
$$

where $\Delta \mathbf{q}_{2}$ is any secondary control law. Introducing this last form in (19), we obtain

$$
\begin{aligned}
V(\mathbf{e}+\boldsymbol{\Delta} \mathbf{e})= & V(\mathbf{e})+\frac{\partial V}{\partial \mathbf{e}} \mathbf{J J}^{+} \boldsymbol{\Delta} \mathbf{e}^{*} \\
& +\frac{1}{2} \boldsymbol{\Delta} \mathbf{e}^{* \top}\left(\mathbf{J J}^{+}\right)^{\top} \frac{\partial V^{2}}{\partial \mathbf{e}^{2}} \mathbf{J J}^{+} \boldsymbol{\Delta} \mathbf{e}^{*}+\frac{\partial V}{\partial \mathbf{e}} \mathbf{J} \boldsymbol{\Delta} \mathbf{q}_{\mathbf{2}} \\
& +\frac{1}{2} \boldsymbol{\Delta} \mathbf{q}_{\mathbf{2}}^{\top} \mathbf{J}^{\top} \frac{\partial^{2} V}{\partial \mathbf{e}^{2}} \mathbf{J} \boldsymbol{\Delta} \mathbf{q}_{2}+\mathcal{O}\left(\|\boldsymbol{\Delta} \mathbf{e}\|^{2}\right) .
\end{aligned}
$$

Since $\mathbf{J J}^{+}=\mathbf{I}$ and using the second order decomposition (19) of $V\left(\mathbf{e}+\Delta \mathbf{e}^{*}\right)$, the condition (18) can be rewritten

$$
\begin{aligned}
V\left(\mathbf{e}+\boldsymbol{\Delta} \mathbf{e}^{*}\right)+\frac{\partial V}{\partial \mathbf{e}} \mathbf{J} \boldsymbol{\Delta} \mathbf{q}_{2} & +\frac{1}{2} \boldsymbol{\Delta} \mathbf{q}_{2}^{\top} \mathbf{J}^{\top} \frac{\partial^{2} V}{\partial \mathbf{e}^{2}} \mathbf{J} \boldsymbol{\Delta} \mathbf{q}_{\mathbf{2}}+\mathcal{O}\left(\|\boldsymbol{\Delta} \mathbf{e}\|^{2}\right) \\
& =V(\mathbf{e}+\boldsymbol{\Delta} \mathbf{e}) \leq V\left(\mathbf{e}+\Delta \mathbf{e}^{*}\right) .
\end{aligned}
$$

For the construction of the control law, the term $\mathcal{O}\left(\|\Delta \mathbf{e}\|^{2}\right)$ is neglected and the condition of application of the secondary term $\mathbf{z}$ is finally reduced to

$$
\frac{\partial V}{\partial \mathbf{e}} \mathbf{J} \boldsymbol{\Delta} \mathbf{q}_{\mathbf{2}}+\frac{1}{2} \boldsymbol{\Delta} \mathbf{q}_{\mathbf{2}}^{\top} \mathbf{J}^{\top} \frac{\partial^{2} V}{\partial \mathbf{e}^{2}} \mathbf{J} \boldsymbol{\Delta} \mathbf{q}_{\mathbf{2}}<0 .
$$

This condition can be compared to the condition (10) obtained in the last section. First of all, if the second-order derivative $\partial^{2} V / \partial \mathbf{e}^{2}$ is neglected, both conditions are equivalent. When the second-order derivative is taken into account, (22) implies a quadratic form of the secondary control law $\Delta q_{2}$. Intuitively, one can see that this quadratic term will lead to a lower bound and a upper bound on the value of $\Delta q_{2}$, which is exactly what is required.

\section{A. Rewriting the Condition}

The inequality (22) is difficult to solve in the general case, since it implies a quadratic term on $\Delta \mathbf{q}_{2}$ weighted by $\partial^{2} V / \partial \mathbf{e}^{2}$. To simplify the computation, we make the assumption that the second-order derivative $\partial^{2} V / \partial \mathbf{e}^{2}$ is the identity. This is the case when considering the norm of the error as a Lyapunov function 


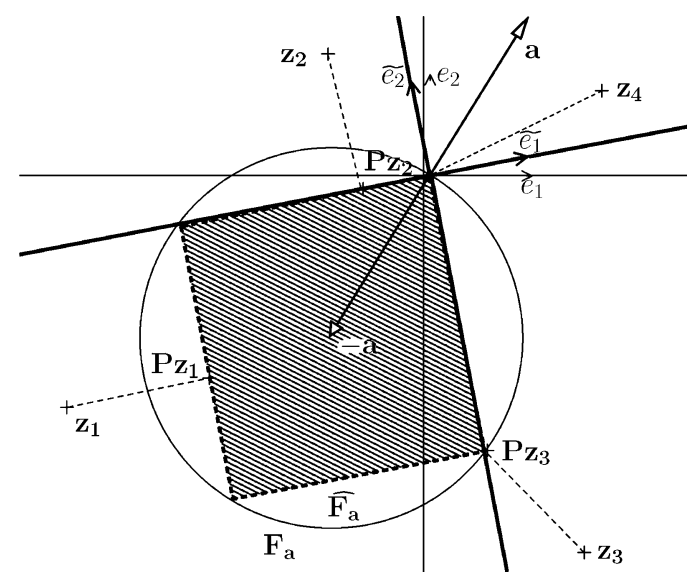

Fig. 2. Two sets $\mathbf{F}_{\mathbf{a}}$ (the circle) and $\widetilde{\mathbf{F}}_{\mathbf{a}}$ (the dashed rectangle) in dimension 2 . The canonical basis of the task space is $\left(e_{1}, e_{2}\right)$. The SVD coordinate frame is $\left(\widetilde{e_{1}}, \widetilde{e_{2}}\right)$. In this basis, the set $\mathbf{F}_{\mathbf{a}}$ is simply a ball of euclidean norm $\|$.$\| , with$ center $-\mathbf{a}$ and radius $\|a\|$, and the set $\widetilde{\mathbf{F}}_{\mathbf{a}}$ is the same parameters ball of norm $\|.\|_{\infty}$. The four points $\mathbf{z}_{1}, \mathbf{z}_{2}, \mathbf{z}_{\mathbf{3}}$ and $\mathbf{z}_{4}$ are projected into $\widetilde{\mathbf{F}}_{\mathbf{a}}$ as a matter of example. Their projection is respectively $\mathbf{P} \mathbf{z}_{1}, \mathbf{P z}_{\mathbf{2}}, \mathbf{P z}_{\mathbf{3}}$ and $\mathbf{0}$. The projection is realized by applying the projection operator computed in Section III-C.

$\left(V=(1 / 2) \mathbf{e}^{\top} \mathbf{e}\right)$, which is a very classical solution. We thus consider the following condition:

$$
\nabla V^{\top} \mathbf{J} \boldsymbol{\Delta} \mathbf{q}_{2}+\frac{1}{2} \boldsymbol{\Delta} \mathbf{q}_{2}^{\top} \mathbf{J}^{\top} \mathbf{J} \boldsymbol{\Delta} \mathbf{q}_{2}<0
$$

where $\nabla V^{\top}=(\partial V / \partial \mathbf{e})\left(\mathbf{e}+\Delta \mathbf{e}^{*}\right)=\left(\mathbf{e}+\Delta \mathbf{e}^{*}\right)^{\top}$.

As in the previous section, the SVD bases are introduced to reduce the complexity to the case where the Jacobian $\mathbf{J}$ is diagonal. We note $\widetilde{\mathbf{e}}=\mathbf{U}^{\top} \mathbf{e}$ and $\widetilde{\mathbf{q}}=\mathbf{V}^{\top} \mathbf{q}$. Condition (23) can be simply written as

$$
\left(\widetilde{\mathbf{e}}+\widetilde{\Delta \mathbf{e}^{*}}\right)^{\top} \boldsymbol{\Sigma} \widetilde{\Delta \mathbf{q}_{2}}+\frac{1}{2}{\widetilde{\Delta \mathbf{q}_{2}}}^{\top} \boldsymbol{\Sigma}^{2} \widetilde{\Delta \mathbf{q}_{2}}<0
$$

By adding the term $(1 / 2)\left\|\widetilde{\mathbf{e}}+\widetilde{\Delta \mathbf{e}^{*}}\right\|^{2}$ to both sides of the inequality, the following factorization is obtained:

$$
\frac{1}{2}\left\|\widetilde{\mathbf{e}}+\widetilde{\Delta \mathbf{e}^{*}}+\Sigma \widetilde{\Delta \mathbf{q}_{2}}\right\|^{2}<\frac{1}{2}\|\widetilde{\mathbf{e}}+\widetilde{\Delta \mathbf{e}}\|^{2} .
$$

\section{B. Construction of the Free Space}

For some vector $\mathbf{a} \neq 0$, let $\mathbf{F}_{\mathbf{a}}$ be the set

$$
\mathbf{F}_{\mathbf{a}}=\{\mathbf{x} \backslash\|\mathbf{a}+\mathbf{x}\|<\|\mathbf{a}\|\} .
$$

$\mathbf{F}_{\mathbf{a}}$ is the ball of radius $\|\mathbf{a}\|$ and center $(-\mathbf{a})$. It is represented on Fig. 2 in the case of a 2-D vector space. Using this definition, the set of all the possible secondary motions $\widetilde{\Delta q_{2}}$ such that $\Delta q$ respects the condition (23) is characterized easily. This condition can thus be written as

$$
\Sigma \widetilde{\Delta \mathbf{q}_{2}} \in \mathbf{F}_{\widetilde{\mathbf{e}}+\widetilde{\Delta e}^{*}}
$$

Given an arbitrary secondary command $\mathbf{z}$, we now want to modify this vector to obtain a second term $\Delta \mathbf{q}_{2}$ that respects this condition. If $\widetilde{\mathbf{z}}$ belongs to the free space $\mathbf{F}_{\widetilde{\mathbf{e}}+\widetilde{\Delta e}^{*}}$, it can be directly added to the primary control law $\left(\Delta \mathbf{q}_{2}=\mathbf{z}\right)$. Otherwise, it has to be projected into the free space. The projection operator is computed using the analytical parametrization of $\mathbf{F}_{\widetilde{\mathbf{e}}+\widetilde{\mathbf{u}_{\mathbf{e}}}}{ }^{*}$. By developing the square of the norms in (25), it is easy to obtain after some simple calculations

$$
2 \sum_{i=1}^{m}\left(\widetilde{e_{i}}+\widetilde{\Delta e_{i}^{*}}\right) \sigma_{i} \widetilde{\Delta q_{2_{i}}}+\sum_{i=1}^{m} \sigma_{i}^{2}{\widetilde{\Delta q_{2_{i}}}}^{2} \leq 0 .
$$

This last condition imposes a decrease of the norm of the error. As in the previous section, we reduce the condition by imposing the decrease of each component of the error. The set $\mathbf{F}_{\widetilde{\mathbf{e}}+\widetilde{\Delta e}}$ is reduced to its Cartesian subset. A sufficient condition is thus

$$
\forall i \in[1 \ldots m], \quad 2\left(\widetilde{e_{i}}+\widetilde{\Delta e_{i}^{*}}\right) \sigma_{i} \widetilde{\Delta q_{2_{i}}}+\sigma_{i}^{2}{\widetilde{\Delta q_{2_{i}}}}^{2}<0 .
$$

The set defined by (29) is noted $\widetilde{\mathbf{F}}_{\widetilde{\mathbf{e}}+\widetilde{\Delta \mathbf{e}}_{\mathbf{e}}}$. It is represented with

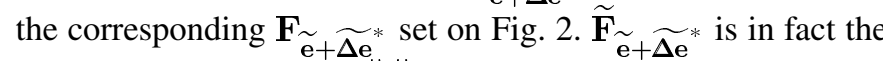
ball defined by the norm $\|\cdot\|_{\infty}$, where

$$
\|\mathbf{x}\|_{\infty}=\max \left\{x_{i}, \quad \mathbf{x}=\left(x_{1}, \ldots, x_{n}\right)\right\} .
$$

\section{Construction of the Projection Operator}

The projection operator into the free space $\widetilde{\mathbf{F}}_{\widetilde{\mathbf{e}}+\widetilde{\Delta \mathbf{e}}} \widetilde{\text { is noted }}$ $\mathbf{P}(\mathbf{z})$. It is a vectorial operator that transforms any vector $\mathbf{z}$ into a secondary control law $\Delta \mathbf{q}_{2}=\mathbf{P}(\mathbf{z})$ such that (29) is respected, and such that the distance $\left(\mathbf{z}, \boldsymbol{\Delta} \mathbf{q}_{2}\right)$ is minimal. Using the analytical characterization of the free space given by (29), this projection operator can be computed component by component within basis $\mathbf{V}$.

Since $\sigma_{i}>0$, (29) can be developed by dividing by $\sigma_{i} \widetilde{\Delta q_{2}}$ (if $\widetilde{\Delta q_{2_{i}}}$ is not zero): $\widetilde{\Delta \mathbf{q}_{2}}$ belongs to the free space of the main task if and only if

$$
\forall i \in[1 \ldots m], \quad \begin{cases} & \widetilde{\Delta q_{2_{i}}}=0 \\ \text { or } & 0<\widetilde{\Delta q_{2_{i}}} \leq-\frac{2}{\sigma_{i}}\left(\widetilde{e_{i}}+\widetilde{\Delta e_{i}^{*}}\right) \\ \text { or } & -\frac{2}{\sigma_{i}}\left(\widetilde{e_{i}}+\widetilde{\Delta e_{i}^{*}}\right) \leq \widetilde{\Delta q_{2_{i}}}<0 .\end{cases}
$$

For each component $\widetilde{z_{i}}$ of $\widetilde{\mathbf{z}}$, the closest $\widetilde{\Delta q_{2}}$ that respects (31) can be computed. By analyzing each case separately, the general expression of $\widetilde{\Delta q_{2_{i}}}$ is deduced

$$
\widetilde{\Delta q_{2_{i}}}= \begin{cases}\widetilde{z_{i}} & \text { if } i>m \text { or } \widetilde{z_{i}}=0 \\ \widetilde{z_{i}} & \text { if } 0<\widetilde{z_{i}} \leq-\frac{2}{\sigma_{i}}\left(\widetilde{e_{i}}+\widetilde{\Delta e_{i}^{*}}\right) \\ \widetilde{z_{i}} & \text { if }-\frac{2}{\sigma_{i}}\left(\widetilde{e_{i}}+\widetilde{\Delta e_{i}^{*}}\right) \leq \widetilde{z_{i}}<0 \\ 0 & \text { if } \sigma_{i} \widetilde{z_{i}}>0 \text { and } \widetilde{e_{i}}+\widetilde{\Delta e_{i}^{*}}<0 \\ 0 & \text { if } \sigma_{i} \widetilde{z_{i}}<0 \text { and } \widetilde{e_{i}}+\widetilde{\Delta e_{i}^{*}}>0 \\ 2\left(\widetilde{e_{i}}+\widetilde{\Delta e_{i}^{*}}\right) & \text { otherwise. }\end{cases}
$$

This equation can be written under a matricial form

$$
\widetilde{\Delta \mathbf{q}_{2}}=\widetilde{\mathbf{P}}(\widetilde{\mathbf{z}})=\widetilde{\mathbf{P}}_{\widetilde{\mathbf{z}}} \widetilde{\mathbf{z}}=\left(\begin{array}{ccc}
p_{1}(\widetilde{\mathbf{z}}) & & 0 \\
& \ddots & \\
0 & & p_{n}(\widetilde{\mathbf{z}})
\end{array}\right) \mathbf{z}
$$




\begin{tabular}{|c|c|c|c|c|}
\hline & $\sigma=0$ & $\begin{array}{r}\left(\widetilde{e_{i}}+\widetilde{\Delta e}\right. \\
z_{i}>-\frac{2}{\sigma_{i}}\left(\widetilde{e_{i}}+\widetilde{\Delta e_{i}^{*}}\right)\end{array}$ & $\begin{array}{l}\sigma_{i} z_{i}<0 \\
z_{i} \leq-\frac{2}{\sigma_{i}}\left(\widetilde{e_{i}}+\widetilde{\Delta e_{i}^{*}}\right)\end{array}$ & $\left(\widetilde{e_{i}}+\widetilde{\Delta e_{i}^{*}}\right) \sigma_{i} z_{i}>0$ \\
\hline Classical & $p_{i}=1$ & $p_{i}=0$ & $p_{i}=0$ & $p_{i}=0$ \\
\hline Directional (15) & $p_{i}=1$ & $p_{i}=1$ & $p_{i}=1$ & $p_{i}=0$ \\
\hline Directional (33) & $p_{i}=1$ & $p_{i}=1$ & $p_{i}=-\frac{2\left(\widetilde{e_{i}}+\widetilde{\Delta e_{i}^{*}}\right)}{\sigma_{i} z_{i}}$ & $p_{i}=0$ \\
\hline
\end{tabular}

Fig. 3. Comparison of the projection operators obtained with the classical redundancy formalism, and with the directional redundancy, first order (15) and second order (33).

where

$$
p_{i}(\widetilde{\mathbf{z}})= \begin{cases}1 & \text { if } i>m \text { or } \widetilde{z_{i}}=0 \\ 1 & \text { if } 0<\widetilde{z_{i}} \leq-\frac{2}{\sigma_{i}}\left(\widetilde{e_{i}}+\widetilde{\Delta e_{i}^{*}}\right) \\ 1 & \text { if }-\frac{2}{\sigma_{i}}\left(\widetilde{e_{i}}+\widetilde{\Delta e_{i}^{*}}\right) \leq \widetilde{z_{i}}<0 \\ 0 & \text { if } \sigma_{i} \widetilde{z_{i}}>0 \text { and } \widetilde{e_{i}}+\widetilde{\Delta e_{i}^{*}}<0 \\ 0 & \text { if } \sigma_{i} \widetilde{z_{i}}<0 \text { and } \widetilde{e_{i}}+\widetilde{\Delta e_{i}^{*}}>0 \\ -\frac{2\left(\widetilde{e_{i}}+\widetilde{\Delta e_{i}^{*}}\right)}{\sigma_{i} \widetilde{z_{i}}} & \text { otherwise. }\end{cases}
$$

As in Section II, the operator $\mathbf{P}$ is not linear: the term $\widetilde{\mathbf{P}_{\mathbf{z}} \mathbf{Z}}$ is thus not linear in $\mathbf{z}$. Moreover, in that case, the matrix $\widetilde{\mathbf{P}_{\mathbf{z}}}$ is not a projection matrix (its diagonal should be composed only of 0 and 1). It is only the matricial form of the non linear projection operator $\mathbf{P}$.

\section{Control Law}

The projection operator $\widetilde{\mathbf{P}_{\mathbf{z}}}$ is computed into the SVD bases $\mathbf{U}$ and $\mathbf{V}$. We note $\mathbf{P}_{\mathbf{z}}$ this operator into the canonical basis of the joint space

$$
\mathbf{P}_{\mathbf{z}}=\widetilde{\mathbf{V P}_{\mathbf{V}^{\top}} \widetilde{\mathbf{z}}} \mathbf{V}^{\top} \text {. }
$$

The control law is finally written. Thanks to the matricial form $\mathbf{P}_{\mathbf{z}}$, the obtained form is very close to the classical redundancy form given in (4)

$$
\Delta \mathbf{q}=\mathbf{J}^{+} \Delta \mathbf{e}^{*}+\mathbf{P}_{\mathbf{z}} \mathbf{z}
$$

where $\mathbf{z}$ is an arbitrary vector, used to perform a secondary task without disturbing the decreasing speed of the main task error.

\section{E. Comparisons and Conclusion}

A comparison between the two projection operators built in Sections II and III with the classical projection operator is given in Fig. 3. As in the classical formalism, the projection operator is used to transform any secondary vector into a secondary control law that does not disturb the main task. Within the same basis $\mathbf{V}$, the projection operator of the classical redundancy is also a diagonal matrix, but whose coefficients are

$$
p_{i}= \begin{cases}1 & \text { if } i>m \\ 0 & \text { otherwise. }\end{cases}
$$

In other terms, the first projection operator (15) that we have defined has more non zero coefficients. When a component of the main task function is not zero, a DOF is freed up. Furthermore, the proposed formalism accelerates the decreasing of each component of the error and takes the secondary task into account in the same way.
Compared to the projection operator (15), the operator (33) induces an alleviation if the secondary control law is too important wrt. the error value. In particular, at task completion, when the error vector is nearly zero, this allows to reduce the effect of the secondary term, and to avoid any oscillation.

\section{Stability OF THE CONTROL LAW}

The definition of the control law (35) and the associate projector lead to the following theorem:

Theorem 4.1: Let $\mathbf{e}$ be any task function whose Jacobian $\mathbf{J}=$ $\partial \mathbf{e} / \partial \mathbf{q}$ is full rank. If the following control law:

$$
\Delta \mathbf{q}=\mathbf{J}^{+} \Delta \mathbf{e}^{*}+\mathbf{P}_{\mathbf{z}} \mathbf{z}
$$

is applied to the robotic system, where:

- $\Delta \mathbf{e}^{*}$ is a stable vector field such that $V=(1 / 2)\|\mathbf{e}\|^{2}$ respects $V\left(\mathbf{e}+\Delta \mathbf{e}^{*}\right)<V(\mathbf{e})$.

- $\mathbf{z}$ is any secondary control law, and $\mathbf{P}_{\mathbf{z}}$ is defined by (33). then, given that $\Delta t$ is sufficiently small, the error e asymptotically converges to zero.

Proof: From (21), it is possible to write

$$
\begin{aligned}
V(\mathbf{e}+\mathbf{J} \boldsymbol{\Delta} \mathbf{q})= & V\left(\mathbf{e}+\Delta \mathbf{e}^{*}\right)+\frac{\partial V}{\partial \mathbf{e}} \mathbf{J P}_{\mathbf{z}} \mathbf{z} \\
& +\frac{1}{2} \mathbf{z}^{\top} \mathbf{P}_{\mathbf{z}}^{\top} \mathbf{J}^{\top} \frac{\partial^{2} V}{\partial \mathbf{e}^{2}} \mathbf{J P}_{\mathbf{z}} \mathbf{z}+\mathcal{O}\left(\|\boldsymbol{\Delta} \mathbf{e}\|^{2}\right) .
\end{aligned}
$$

By construction of $\mathbf{P}_{\mathbf{z}}$, we know that $\Delta \mathbf{q}_{\mathbf{2}}=\mathbf{P}_{\mathrm{z}} \mathbf{z}$ respects (22). Introducing (22) in the previous equation, we obtain

$$
V(\mathbf{e}+\mathbf{J} \boldsymbol{\Delta} \mathbf{q})<V\left(\mathbf{e}+\boldsymbol{\Delta} \mathbf{e}^{*}\right)+\mathcal{O}\left(\|\boldsymbol{\Delta} \mathbf{e}\|^{2}\right) .
$$

Subtracting $V(\mathbf{e})$ from both side of the inequality finally gives

$$
\Delta V<\Delta V^{*}+\mathcal{O}\left(\|\Delta \mathbf{e}\|^{2}\right)
$$

where $\Delta V=V(\mathbf{e}+\mathbf{J} \boldsymbol{\Delta} \mathbf{q})-V(\mathbf{e})$ and $\Delta V^{*}=V\left(\mathbf{e}+\Delta \mathbf{e}^{*}\right)-$ $V(\mathbf{e})$. It is thus possible to find $\Delta \mathbf{e}$ small enough to have $\Delta V \leq$ $\Delta V^{*}$. Since $\Delta \mathrm{e}$ has the same order as $\Delta t$ and given that $\Delta t$ is sufficiently small, then $\Delta V \leq \Delta V^{*}$. By definition of $\Delta \mathrm{e}^{*}$, $\Delta V^{*}$ is negative, which proves that $V$ is a correct Lyapunov function for control law (37).

Remark 1: We have not been able to determine any theoretical value of $\Delta t$ to ensure the stability for any large value of z. In practice, the value of $\Delta t$ used to compute the control law could be chosen smaller than the actual value of the sampling interval of the control input. This choice allows ensuring the practical stability of the system when $\mathbf{z}$ is large. However, we have not used the possibility to tune this parameter in the experiments presented in the following: $\Delta t$ has been chosen equal 
to the system time interval. No unstability have been noticed during the experimental setup.

Remark 2: Theorem 4.1 supposes that the desired evolution $\Delta \mathbf{e}^{*}$ is such that $\Delta V^{*}$ is negative. When $\Delta \mathbf{e}^{*}=-\lambda \mathbf{e} \Delta t$ as classically done, this last hypothesis is simply obtained by choosing the gain $\lambda$ and the step size $\Delta t$ small enough.

Theorem 4.1 only gives the asymptotic convergence of the main task. It does not say anything about the secondary task. Since the main task has priority, the convergence of the secondary task can not be ensured in the general case. However, it is expected to obtain at least the convergence of the part of the secondary task located inside the null space of the main task, that is to say

$$
\mathbf{P}_{\dot{\mathbf{q}}_{2}} \dot{\mathbf{q}}_{2}=0 \text {. }
$$

In the general case where the secondary task can be any $n$-dimensional vector, nothing can be proved. We thus limit the stability study to the classical case where the secondary task is the gradient of a cost function $h$ to be minimized [13], [17]. Indeed, the secondary tasks used to experiment the control law on the robot are derived from a cost function (see Section V-B). Using Theorem 4.1, it is easy to deduce the asymptotic convergence to a region where the secondary task gradient is in the null space of the main task, that is to say the cost function $h$ is asymptotically minimized under the constraint $\mathbf{e}=\mathbf{0}$.

Corollary 4.1: Let $h$ be any positive convex function defined on the joint space. Using the hypotheses of Theorem 4.1, if the control law (37) is applied to the robotic system, with $\mathbf{z}=-\nabla_{\mathbf{q}}^{\top} h$, then the cost function $h$ is asymptotically minimized under the constraint $\mathbf{e}=\mathbf{0}$.

Proof: The control law is asymptotically equivalent to (from Theorem 4.1)

$$
\dot{\mathbf{q}}=-\mathbf{P}_{-\nabla_{\mathbf{q}}^{\top} \mathbf{h}} \nabla_{\mathbf{q}}^{\top} h .
$$

We assume that $h$ does not depend of the independent time variable

$$
\dot{h}=\frac{\partial h}{\partial \mathbf{q}} \dot{\mathbf{q}}=\nabla_{\mathbf{q}}^{\top} h \dot{\mathbf{q}} .
$$

By introducing (42) in (43), we obtain

$$
\dot{h}=-\nabla_{\mathbf{q}}^{\top} h \mathbf{P}_{-\nabla_{\mathbf{q}}^{\top} \mathbf{h}} \nabla_{\mathbf{q}}^{\top} h .
$$

Since $\mathbf{P}_{\mathbf{z}}$ is definite non-negative for any vector $\mathbf{z}$ (due to the form (33) of the coefficients of the equivalent diagonal matrix $\widetilde{\mathbf{P}_{\widetilde{\mathbf{z}}}}$, we finally obtain

$$
\dot{h} \leq 0 .
$$

This result is sufficient to prove the stability of the secondary criterion, but does not prove that the second criterion is globally asymptotically minimized ( $\dot{h}$ should be negative, and it is only non-positive). However, it proves that $h$ is stable and decreases until $\mathbf{P}_{-\nabla_{\mathbf{q}}^{\top} \mathbf{h}} \nabla_{\mathbf{q}}^{\top} h$ becomes null, that is the minimum under the constraint $\mathbf{e}=\mathbf{0}$ is reached.

A similar Lyapunov function can be given for the control scheme using the classical redundancy formalism. Let $h_{c l a}$ be the secondary cost function value over time using the classical redundancy formalism scheme, and let $h_{d i r}$ be the secondary cost function value using the scheme proposed in Theorem 4.1 Through the misuse of notation $\|\mathbf{x}\|_{\mathbf{P}}=\mathbf{x}^{\top} \mathbf{P} \mathbf{x}$, we can write

$$
\begin{aligned}
& \dot{h_{c l a}}=-\left\|\nabla_{\mathbf{q}}^{\top}\right\|_{\mathbf{P}} \\
& \dot{h_{d i r}}=-\left\|\nabla_{\mathbf{q}}^{\top}\right\|_{\mathbf{P}_{-\nabla_{\mathbf{q}}^{\top}}} .
\end{aligned}
$$

Since the singular values of $\mathbf{P}_{-\nabla_{\mathrm{q}}^{\top}}$ are all greater than or equal to the singular values of $\mathbf{P}$ (due to (33) and (36)), the following inequality can be written:

$$
\dot{h_{\text {dir }}} \leq \dot{h_{\text {cla }}} .
$$

The cost function converges to similar local minima using both schemes. However, this last inequality proves that the convergence is faster using the directional redundancy.

To conclude, we have shown in this section that the global minimization of $h$ is of course not necessarily ensured. The system converges to a local minimum imposed by the constraint $\mathbf{e}=\mathbf{0}$ as expected. However Theorem 4.1 and Corollary 4.1 prove that the system is globally stable, and asymptotically converges to the main task completion and to the best reachable local minimum of the secondary task.

\section{Application to Visual Servoing}

All the work presented above has been realized under the only hypothesis that the main task $\mathbf{e}$ is a task function as described in [21]. The method is thus fully general and can be applied for numerous sensor-based closed-loop control problems. For this article, the method has been applied to visual servoing. In the experiments described in the next section, the robot has to move with respect to a visual target, and simultaneously to take into account a secondary control law. For the simulations, this secondary term was simply an arbitrary velocity. For the experiments on the real robot, the joint limits and possible occlusions were considered. In this section, the classical visual servoing formalism is first quickly recalled (Section V-A). Two avoidance laws are then presented for joint-limit and visual-occlusion avoidance, based on the gradient of a cost function [13], [15], [17]. We have chosen to use a solution proposed from path planning [18] which ensures an optimal computation of the gradient by the use of a pseudo inverse. This general method is presented in Section V-B and the two cost functions are given in Section V-C.

\section{A. Main Task Function Using Visual Servoing}

In the experiments presented below, an eye-in-hand robot has to move with respect to a visual target (a sphere in simulation and a rectangle composed of four points easily detectable for the experiments on a real robot). By choosing a very simple target, the experiments have focused on the control part of the work.

The task function e used in the following is defined as the difference between the visual features $\mathbf{s}$ computed at the current time and the visual features $\mathbf{s}^{*}$ extracted from the desired image [7], [12]:

$$
\mathrm{e}=\mathbf{s}-\mathbf{s}^{*} \text {. }
$$

The interaction matrix $\mathbf{L}_{\mathbf{s}}$ related to $\mathbf{s}$ is defined such that $\dot{\mathbf{s}}=$ $\mathbf{L}_{\mathbf{s}} \mathbf{v}$, where $\mathbf{v}$ is the camera instantaneous velocity. From (49), 
it is clear that the interaction matrix $\mathbf{L}_{\mathbf{s}}$ and the task Jacobian $\mathbf{J}$ are linked by the relation

$$
\mathbf{J}=\mathbf{L}_{\mathbf{s}} \mathbf{M} \mathbf{J}_{\mathbf{q}}
$$

where the matrix $\mathbf{J}_{\mathbf{q}}$ denotes the robot Jacobian $\left(\dot{\mathbf{r}}=\mathbf{J}_{\mathbf{q}} \dot{\mathbf{q}}\right)$ and $\mathbf{M}$ is the matrix that relates the camera instantaneous velocity $\mathbf{v}$ to the variation of the chosen camera pose parametrization $\mathbf{r}(\mathbf{v}=\mathbf{M} \dot{\mathbf{r}})$. The classical proportional control law $\dot{\mathbf{e}}^{*}=-\lambda \mathbf{e}$ was used. The control law finally used in the experiment is then

$$
\dot{\mathbf{q}}=-\lambda\left(\mathbf{L}_{\mathbf{s}} \mathbf{M} \mathbf{J}_{\mathbf{q}}\right)^{+}\left(\mathbf{s}-\mathbf{s}^{*}\right)+\mathbf{P}_{\mathbf{z}} \mathbf{z}
$$

where $\mathbf{z}$ can be any vector used to realize a secondary task.

In the experiments presented below, the target projection in the image is a continuous region $\mathcal{O}$ (an ellipse in simulation, a quadrilateral on the real robot). In order to have a better and easier control over the robot trajectory, six approximatively decoupled visual features are chosen as proposed in [23].

The two first features are the position $x_{g}$ and $y_{g}$ of the center of gravity, controlling the target centering. The third feature $a_{n}$ controls the distance between the camera and the target. It is based on the area of the object in the image. The fourth feature $\alpha$ is defined as the orientation of the object in the image and is mainly linked to the rotational motion around the optical axis. The two last features $s_{x}$ and $s_{y}$ are defined from third order moments to decouple the translational velocities $v_{x}$ and $v_{y}$ from their corresponding rotational velocities $\omega_{y}$ and $\omega_{x}$. The reader is invited to refer to [23] for more details and for the analytical form of the interaction matrix of the chosen visual features.

For the experiments on the real robot, the observed region is the image of a rectangle. The six features can thus be used to control the six DOF of the robot. For the second experiment on the robot however, only the four first features are used, to introduce some redundancy in the control system. For the experiments in simulation, the ellipse region is the image of a sphere. Only the three first feature can be used, and control three DOF of the robot.

\section{B. Cost Function for Avoidance}

The secondary task can be used to minimize the constraints imposed by the environment. The constraints are described by a cost function. The gradient $\mathbf{g}(\mathbf{q})$ of this cost function can be considered as an artificial force, pushing the robot away from the undesirable configurations.

The cost function is expressed directly in the space of the configuration to avoid (e.g. the cost function of visual-occlusion constraint is expressed in the image space). Let $\boldsymbol{\Phi}$ be a parametrization of this space. The cost function can be written $V_{\boldsymbol{\Phi}}=V(\boldsymbol{\Phi}(\mathbf{q}))$. The optimal corresponding artificial force is proved to be [18]

$$
\mathbf{g}_{\boldsymbol{\Phi}}(\mathbf{q})=-\left(\frac{\partial \Phi}{\partial \mathbf{q}}\right)^{+} \nabla_{\boldsymbol{\Phi}}^{\top} V_{\boldsymbol{\Phi}} .
$$

Note the use of the Jacobian pseudo inverse in the final artificial force formulation. Classical methods propose generally to use simply the transpose of the Jacobian, the artificial force being then $\mathbf{g}_{\boldsymbol{\Phi}}(\mathbf{q})=-(\partial \boldsymbol{\Phi} / \partial \mathbf{q})^{\top} \nabla_{\boldsymbol{\Phi}}^{\top} V_{\boldsymbol{\Phi}}$. Since the pseudo inverse provides the least-square solution, the resulting artificial force (52) is the most efficient one at equivalent norm.

\section{Occlusion and Joint-Limit Avoidance Laws}

For each kind of constraint, an avoidance control law can now be computed by simply defining an associate cost function. In this section, we present two cost functions, the first one for the joint-limit avoidance, the second one for the occlusion avoidance. The obtained control laws are also explicitly written.

1) Joint-Limit Avoidance: The cost function $V_{\mathbf{q}}^{\text {joint }}$ is defined directly in the joint space (the Jacobian $\partial \Phi / \partial \mathbf{q}$ defined in (52) is thus the identity matrix). It reaches its maximal value near the robot joint limits, and the gradient is nearly zero far from the limits.

The robot lower and upper joint limits for each axis $i$ are denoted $\bar{q}_{i}^{\min }$ and $\bar{q}_{i}^{\max }$. The robot configuration $\mathbf{q}$ is acceptable if for each $i, q_{i} \in\left[\bar{q}_{\ell i}^{\min }, \bar{q}_{\ell i}^{\max }\right]$, where

$$
\left\{\begin{array}{l}
\bar{q}_{\ell_{i}}^{\min }=\bar{q}_{i}^{\min }+\rho \Delta \bar{q}_{i} \\
\bar{q}_{\ell i}^{\max }=\bar{q}_{i}^{\max }-\rho \Delta \bar{q}_{i}
\end{array}\right.
$$

where $\Delta \bar{q}_{i}=\bar{q}_{i}^{\max }-\bar{q}_{i}^{\min }$ is the length of the domain of the articulation $i$, and $\rho$ is a tuning parameter, in $[0,1 / 2]$ (typically, $\rho=0.1) . \bar{q}_{\ell i}^{\min }$ and $\bar{q}_{\ell i}^{\max }$ are activation thresholds. In the acceptable interval, the avoidance force should be zero. The cost function is defined by [5]

$$
V_{\mathbf{q}}^{\text {joint }}(\mathbf{q})=\frac{1}{2} \sum_{i=1}^{n} \frac{\Delta_{i}^{2}}{\Delta \bar{q}_{i}}
$$

where

$$
\Delta_{i}=\left\{\begin{array}{ll}
q_{i}-\bar{q}_{\ell_{i}}^{\min }, & \text { if } q_{i}<\bar{q}_{\ell_{i}}^{\min } \\
q_{i}-\bar{q}_{\ell i}^{\max }, & \text { if } q_{i}>\bar{q}_{\ell i}^{\max } \\
0, & \text { otherwise }
\end{array} .\right.
$$

According to (52), the artificial force for avoiding the joint limits is

$$
\mathbf{g}_{\mathbf{q}}^{\text {joint }}=-\left(\frac{\partial \mathbf{q}}{\partial \mathbf{q}}\right)^{+} \nabla_{\mathbf{q}}^{\top} V_{\mathbf{q}}^{\text {joint }}=-\nabla_{\mathbf{q}}^{\top} V_{\mathbf{q}}^{\text {joint }} .
$$

2) Occlusion Avoidance: Occlusion avoidance depends on data extracted from the image. An image processing step detects the occluding object (if any). The avoidance law should maximize the distance $d$ between the occluding object and the visual target that is used for the main task. Let $d_{x}$ and $d_{y}$ be the $x$ and $y$ coordinates of the distance between the target and the occluding object $\left(d=\sqrt{d_{x}^{2}+d_{y}^{2}}\right)$ and $\mathbf{x}_{\mathbf{a}}$ be the point of the occluding object that is the closest to the target.

The cost function $V_{\mathbf{s}}^{o c c}$ is defined directly in the image space. It is maximal when $d$ is 0 , and nearly 0 when $d$ is high. Like in [17], we simply choose

$$
V_{\mathbf{s}}^{o c c}(d)=e^{-\beta d^{2}}
$$

where $\mathbf{s}=(x, y)$ denotes the image parameters. The parameter $\beta$ is arbitrary and can be used to tune the effect of the avoidance control law. The gradient in the image space is obtained by a simple calculation

$$
\nabla_{\mathbf{s}} V_{\mathbf{s}}^{o c c}=\left(\begin{array}{l}
-2 \beta d_{\mathbf{x}} e^{-\beta d^{2}} \\
-2 \beta d_{y} e^{-\beta d^{2}}
\end{array}\right)
$$




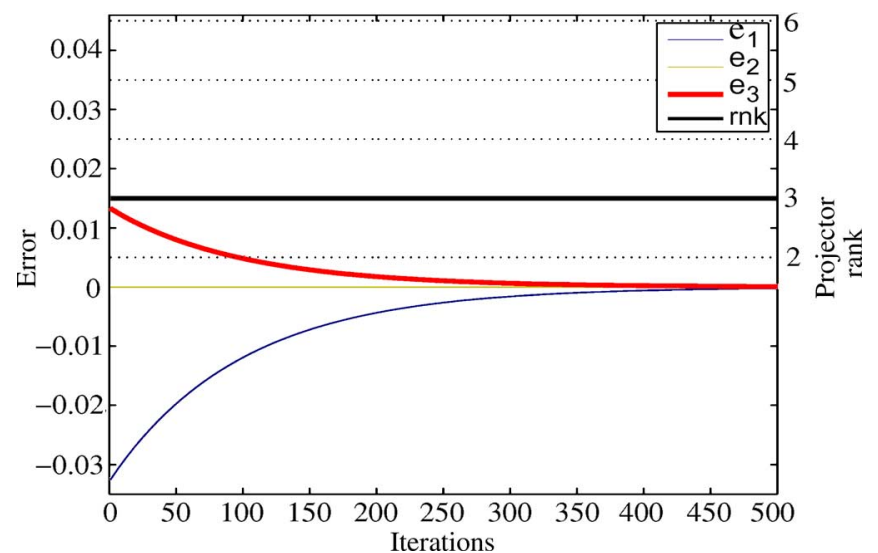

Fig. 4. Experiment A: main task error and projection operator rank using the classical redundancy formalism. The projection operator rank is always 3 . The main task error is not modified by the secondary task: it is a perfect exponential decrease.

The artificial force that avoids the occlusions can be now computed using (52). The transformation from the image space to the joint space is given by

$$
\mathbf{g}_{\mathbf{s}}=-\left(\frac{\partial \mathbf{s}}{\partial \mathbf{r}} \frac{\partial \mathbf{r}}{\partial \mathbf{q}}\right)^{+} \nabla_{\mathbf{s}} V_{\mathbf{s}}^{o c c}=-\left(\mathbf{L}_{\mathbf{x}} \mathbf{M} \mathbf{J}_{\mathbf{q}}\right)^{+} \nabla_{\mathbf{s}} V_{\mathbf{s}}^{o c c}
$$

where $\mathbf{M}$ and $\mathbf{J}_{\mathbf{q}}$ are the transformation matrices defined in (50), and $\mathbf{L}_{\mathbf{x}}$ is the well-known interaction matrix related to the image point $\mathbf{x}_{\mathbf{a}}$ [12].

\section{EXPERIMENTAL RESULTS}

Three different experiments have been realized to highlight the advantages of our method. The first one has been realized in simulation to study in detail the differences between classical redundancy and directional redundancy. The two others experiments have been realized with a real robot, the first one with all DOF constrained by the main task (redundancy is available only through the directional redundancy framework), the second one with four of the six robot DOF constrained by the main task.

\section{A. Experiments in Simulation}

The first experiments have been realized in simulation. It was thus possible to control all the parameters of the experiment to study the control law in depth. In particular, the sensor noise was easy to tune. In this experiment, the robot has to position with respect to a sphere. The main task is composed of three features: center of gravity $\left(x_{g}, y_{g}\right)$ and sphere area $\left(a_{n}\right)$ in the image. Three DOF remain free using the classical redundancy formalism. The secondary task is simply a displacement along the $\mathrm{X}$-axis of the camera

$$
\begin{aligned}
\mathbf{e}_{\text {main }} & =\left(x_{g}, y_{g}, a_{n}\right) \\
\mathbf{e}_{\text {sec }} & =(v, 0,0,0,0,0) .
\end{aligned}
$$

The first part of the experiment was realized without any noise in the measures. A summary is provided in Figs. 4-7. The second part studies the effects of the sensor noise on the control

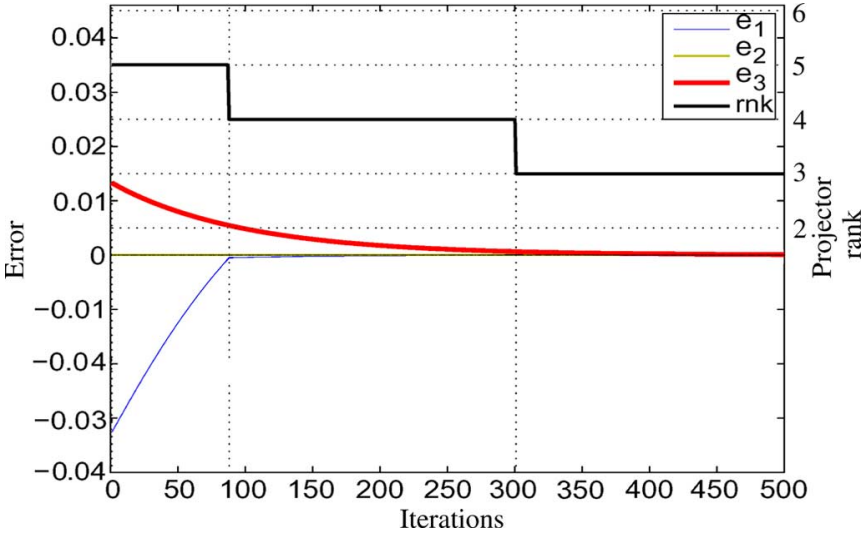

Fig. 5. Experiment A: main task error and projection operator rank using the directional redundancy formalism. The rank of the projection operator is not constant during the servo. At the beginning of the servo, the DOF corresponding to Components $e_{1}$ and $e_{3}$ are available for the secondary task. The projection operator rank is thus 5 . The DOF corresponding to $e_{1}$ is used by the secondary task. The decrease is thus faster than required by the main task. When $e_{1}$ becomes null, the projection operator rank decreases (Iteration 80 ). The DOF corresponding to $e_{3}$ is available until Iteration 300 . At this instant, the main task error is null. No additional DOF remains free. The projection operator is thus the same than the one obtained by the classical formalism.

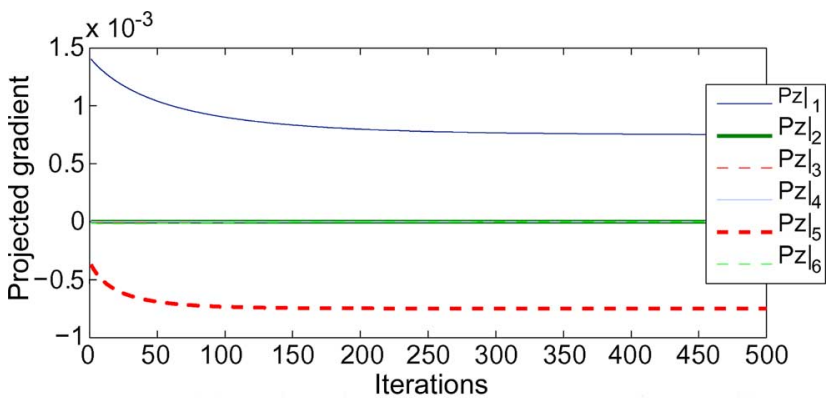

(a)

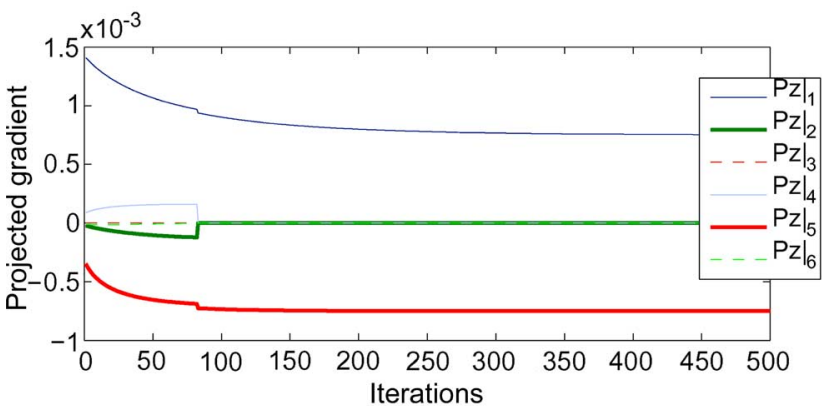

(b)

Fig. 6. Experiment A: comparison of the projected secondary task using the classical and the directional redundancy formalism. While the first component of the main task is not null (until Iteration 80), two components of the secondary tasks are taken into account using the directional redundancy formalism, but nullified by the classical formalism. (a) Classical redundancy formalism; (b) directional redundancy formalism.

law, and proposes a simple hysteresis comparator to filter the noise when computing the projection operator.

1) Comparison With the Classical Redundancy Formalism: Using the classical redundancy formalism, the projection-operator rank is 3 during the whole execution (see Fig. 4). The error behavior is a perfect exponential decrease, as specified by $\dot{\mathrm{e}}^{*}$. 


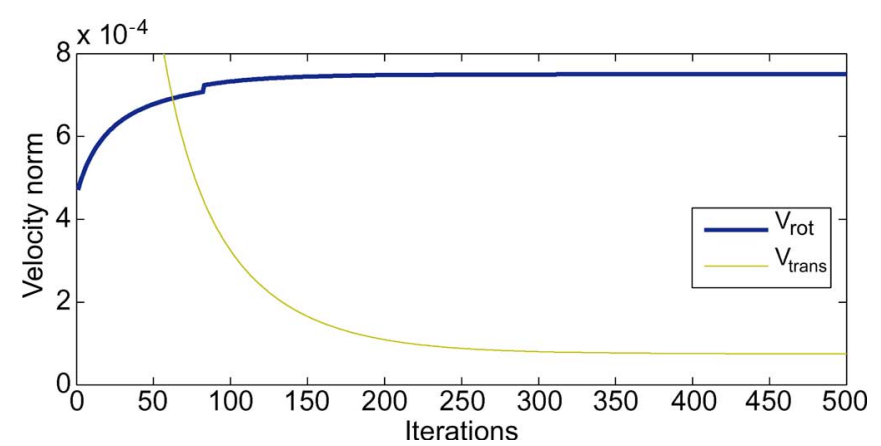

Fig. 7. Experiment A: control law using the directional redundancy formalism. The norm of the translational and rotational velocities are represented. The velocity changes at Iteration 80 , corresponding to the projection-operator rank decrease (see Fig. 5).

On the contrary, when using the formalism proposed above, the projection-operator rank is greater than 3 while the error of the main task is not null. As can be seen on Fig. 5, the convergence of the first component of the main-task error is accelerated by the secondary task. When it reaches 0 , the projection operator looses a rank. The third component of the error does not correspond to any part of the secondary task, and is thus let untouched. The corresponding DOF is available but not used by the secondary task. When $e_{3}$ reaches 0 , the projection operator looses another rank. From this point, there is no difference at all between the two redundancy formalisms: the two projection operators are equal, and the robot behavior is the same.

The effect of the projection operator on the secondary task is shown in Fig. 6. While Component $e_{3}$ is not null, a part of the secondary task is nullified by the classical projection operator, but taken into account by the proposed control law. Intuitively, the main task is composed of two parts: centering and zooming. Since the secondary task is a translation along $\mathrm{X}$-axis, it modifies the centering. When the main task is realized, the projection operator mainly generates an artificial rotation $\omega_{y}$ to compensate the secondary velocity $v_{x}$ and thus preserves the centering. In the initial configuration presented here, the secondary task has an acceptable influence on the centering, since it helps to realize the centering. It is thus accepted when using the directional redundancy formalism while the object is not centered in the image. As soon as the centering is realized, the part of the secondary task that modifies the centering is rejected, and nullified by the projection operator. Component $e_{1}$ which is part of the centering converges thus faster than using the classical redundancy formalism. The DOF corresponding to the zoom is also available using the directional redundancy. However, since the secondary task has no effect on the zoom, it is not used and Component $e_{3}$ of the main task which corresponds to the zoom converges like when using the classical formalism.

2) In Presence of Noise: A white noise is added directly to the computation of the main-task error. The error will thus never reach 0 . The task is considered to be completed when it is smaller than a threshold equals to the variance of the noise. The computation of the projection operator also requires to test if the components of the error is null: a DOF is available when the corresponding error component is not null but disappears

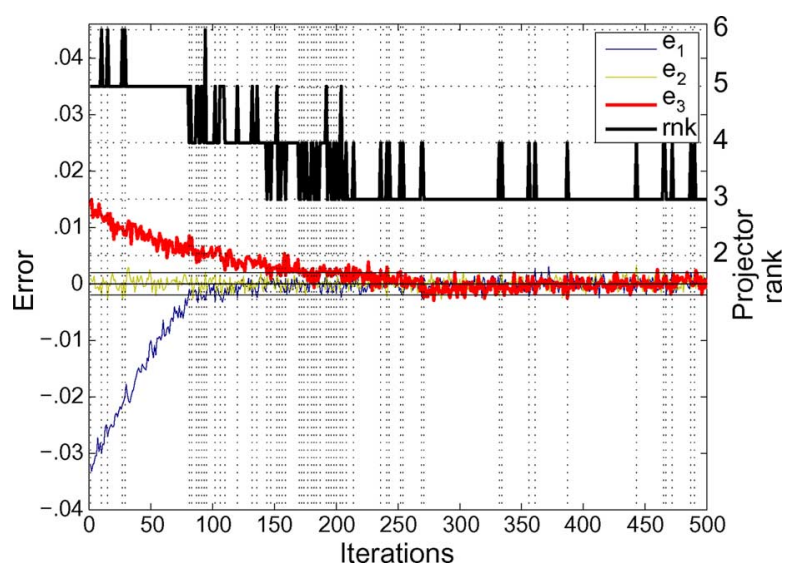

Fig. 8. Experiment A: main task error and projection operator rank using the directional redundancy formalism in the presence of noise without any hysteresis comparator. The projection operator rank increases each time an error component passes through the threshold.

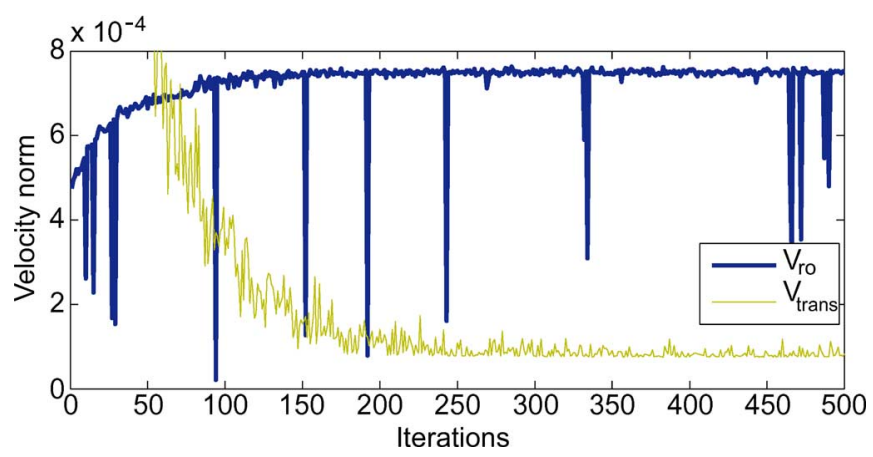

Fig. 9. Experiment A: control law using the directional redundancy formalism in the presence of noise without any hysteresis comparator. A peak appears each time the projection operator rank increases.

as soon as the component reaches 0 (see (33)). Once again, the error component is considered null if it is smaller than the noise variance.

The main task error and the rank of the projection operator are given in Fig. 8. As can be seen on this figure, the rank of the operator is very noisy. Since the rank is discrete, the white noise is amplified when computing the projection operator and induces thus a very strong perturbation: each time the error increases above the threshold because of the noise, the projection operator rank increases. As can be seen on Fig. 9, the noise is amplified by the control law computation and a lot of strong discontinuities appear in the control law.

3) Hysteresis Comparator: The problem is solved using a simple principle known as the Schmitt trigger in electrical engineering [22]. Two thresholds are used to determine if the error is null. The output of the comparison is not null if the error is greater than the higher threshold; the output is null if the error is smaller than the lower threshold. And when the error is between the two thresholds, the output retains the previous value. The lower threshold is set to the noise variance, which corresponds to one standard deviation. The higher threshold has to be set so that an error greater than the threshold has a very low probability to be only due to noise. We have set it to three standard deviations of the noise, which correspond 


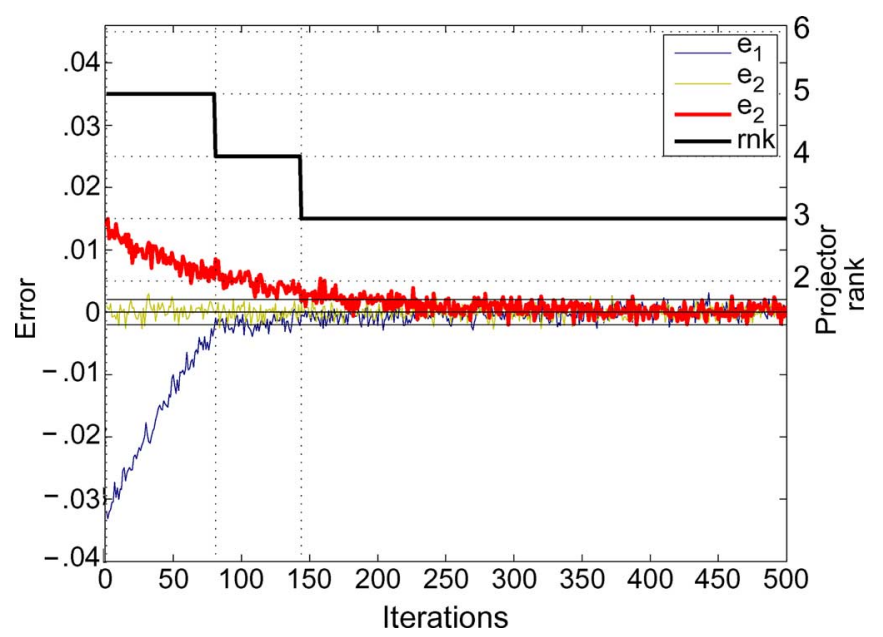

Fig. 10. Experiment A: main task error and projection operator rank using the directional redundancy formalism in the presence of noise using an hysteresis comparator. When an error component becomes less than the first threshold, the projection operator decreases. It will increase only if the corresponding error component increases above a second higher threshold. The projection operator is not noisy any more. Like in the non-noisy experiment, it decreases a first time when $e_{1}$ becomes null (Iteration 70) and a second time when $e_{3}$ becomes null (Iteration 140).

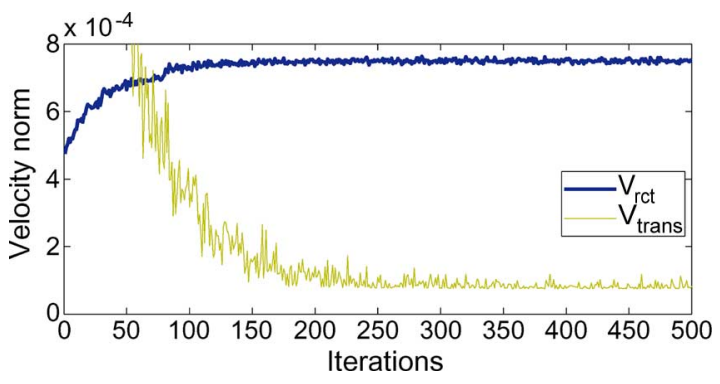

Fig. 11. Experiment A: control law using the directional redundancy formalism in the presence of noise using an hysteresis comparator. The control law is not discontinuous any more. The noise in the control law is due to the noise in the sensor measures, and is equivalent to the noise that we have set in input. The control law computation does not amplify the noise.

numerically to a probability of false detection approximately equals to $99.7 \%$.

The results of the simulation using the hysteresis system are presented in Figs. 10-12. The projection operator rank is not noisy any more (see Fig. 10). The noise in the control law corresponds to the noise on the error: the signal/noise ratio is no more amplified by the projection computation (see Fig. 11). The projected secondary task is given in Fig. 12. The values are very similar to the ones obtained without any noise (given on Fig. 6(b)). This emphasizes that the hysteresis comparator has removed the major part of the noise when computing the projection operator.

In the following real experiments, the hysteresis comparator has also been used. The two thresholds could be set by a fastidious analysis of the probability distribution of the sensor measures (the image processing in our case). We have assumed a Gaussian distribution. The variance has then been computed from experimental results directly and the same two thresholds as mentioned above have been used.

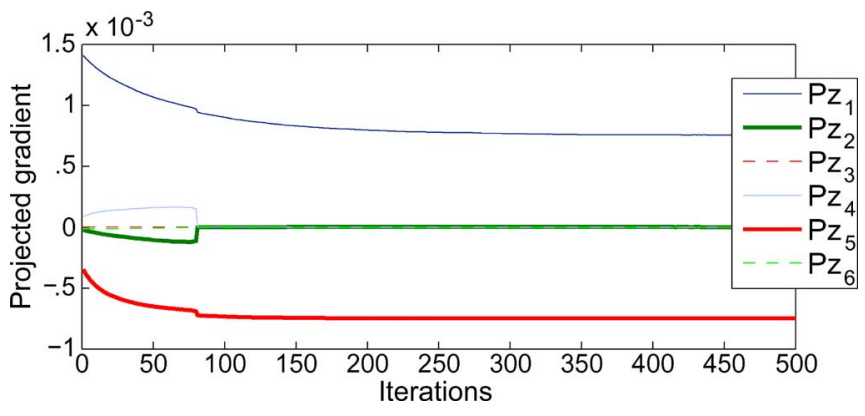

Fig. 12. Experiment A: projected secondary task using the directional redundancy formalism in the presence of noise using an hysteresis comparator. The projected secondary task is very similar to the one obtained without noise (see Fig. 6(b)). The projection operator is thus robust to the noise in the sensor measure.

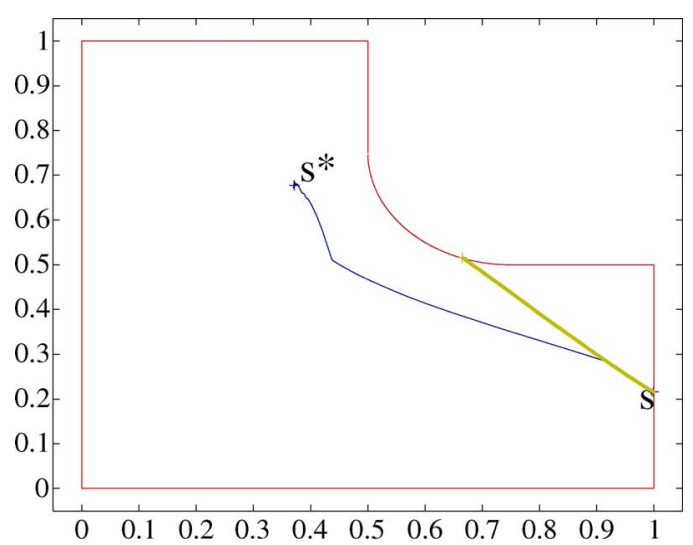

Fig. 13. Experiment B: joint trajectories of the two first components of the joint vector. It corresponds mainly to the camera position in the plane $X Y$. The joint limits are represented in red. The trajectory with the classical redundancy formalism is represented in yellow. It ends in the joint limits. The trajectory with the proposed method is in blue. The positioning task succeeds ( $\mathbf{s}^{*}$ is reached).

\section{B. First Experiment on the Robot (Six DOF Constrained)}

The two next experiments have been realized on a six-DOF eye-in-hand robot. In this experiment, the robot has to reach a unique pose with respect to the visual target. The main task uses all the DOF of the robot. The projection operator computed using the classical redundancy formalism is thus null.

Thanks to the choice of adequate visual features, the camera trajectory without any secondary task is almost a straight line (it is not a perfect straight line because the features are not perfectly decoupled). Since the robot joint domain is not convex and the trajectory is close to a straight line, the robot reaches its joint limits during the servo. Since there is no DOF left using the classical redundancy formalism, the main task fails when the robot reaches its joint limits as shown in Fig. 13.

Using the method proposed above, the projection operator is not null as long as the error of the main task is not zero. Fig. 14 gives the rank of the projection matrix $\mathbf{P}_{\mathbf{z}}$ during the execution. When the robot is near the joint limits, the projection operator is not null and the projected gradient is also not null (see Fig. 15). The induced secondary control law is large enough to modify the trajectory imposed by the main task and to avoid the joint limits. Fig. 16 presents the evolution of three visual features 


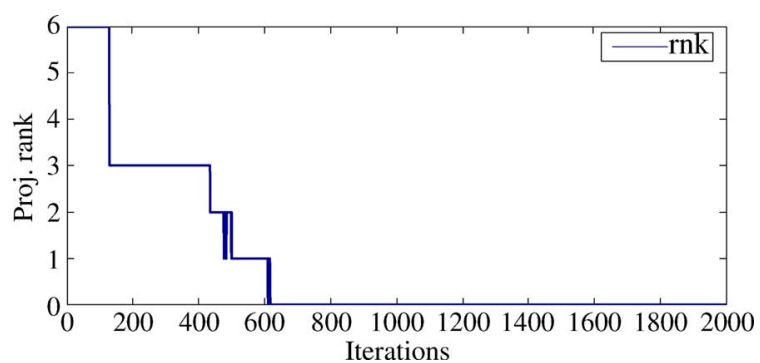

Fig. 14. Experiment B: rank of the projection operator computed using the proposed approach during the servo.
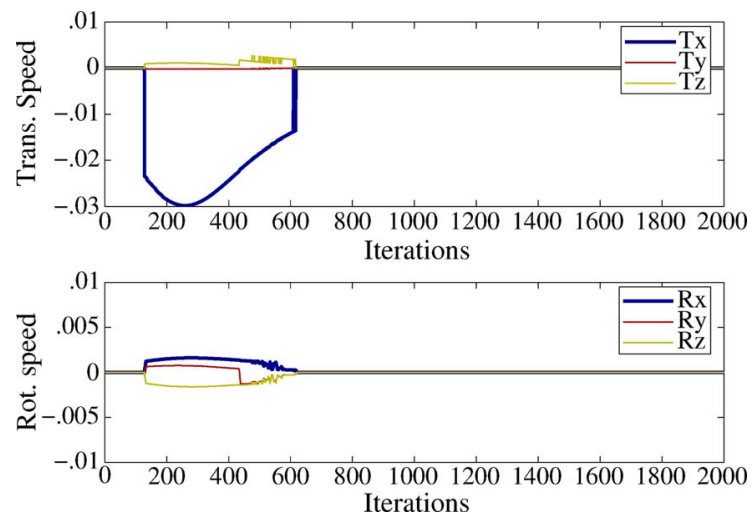

Fig. 15. Experiment B: projected gradient using the proposed method. The secondary control law mainly increases the third component of the joint speed, that corresponds to the speed along the optical axis.

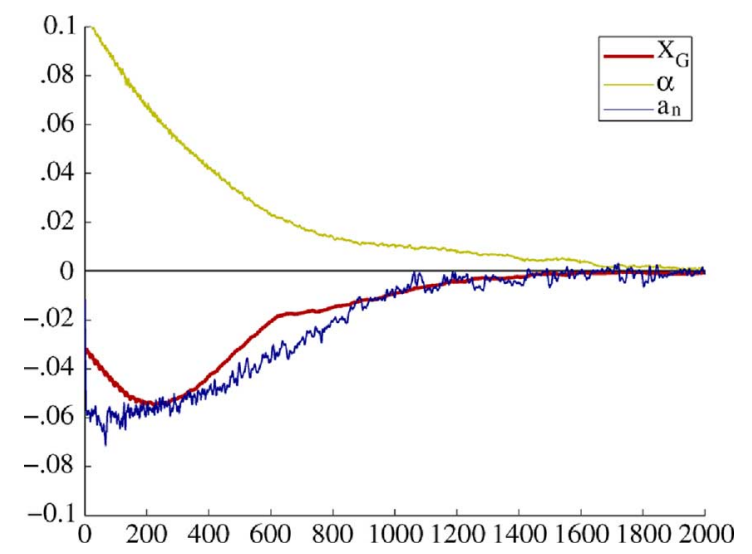

Fig. 16. Experiment B: evolution of the visual features when applying the proposed method. The two features $x_{g}$ and $a_{n}$ (plotted in red and blue) are modified by the avoidance law. On the opposite, feature $s_{y}$ (plotted in green) is not modified.

whose value is modified by the secondary control law. The projection operator mainly accelerates the decreasing speed of features $x_{g}$ and $a_{n}$ controlling the centering (pan) and the motion along the optical axis. Using the framework presented above, it is thus possible to free up some additional DOF that are not available within the classical redundancy formalism. The main task is correctly completed, and the servo is not slowed by the secondary control law.

\section{Second Experiment on the Robot (Four DOF Constrained)}

In the previous experiment, no avoidance law could be taken into account by the classical redundancy formalism. It was thus
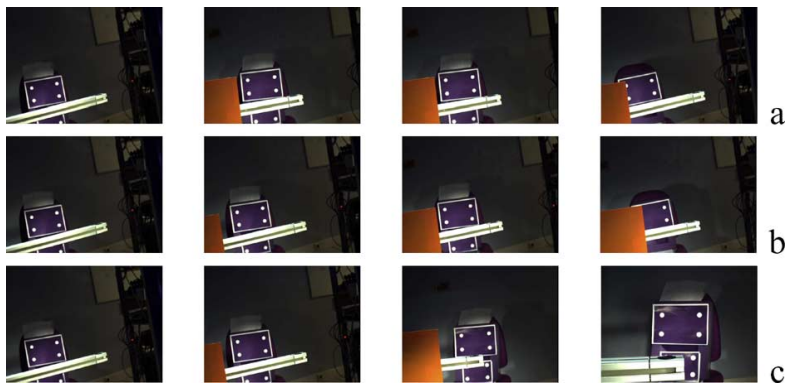

Fig. 17. Experiment C: main phases of the servo (a) without avoidance, (b) with the avoidance law projected using the classical redundancy formalism, and (c) using the directional redundancy. The pictures are taken by the embedded camera. The visual target is the four-white-points rectangle. The occluding object is the orange shape that appears in the left of the image.

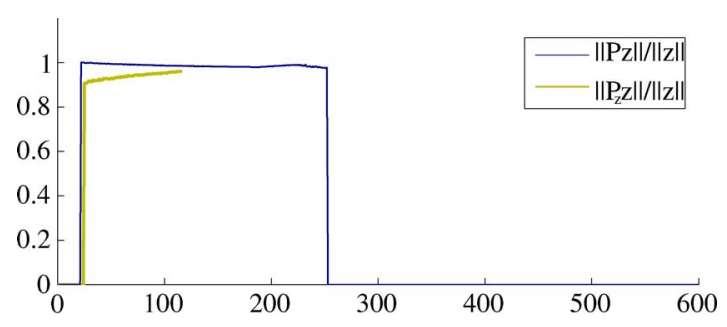

Fig. 18. Experiment C: norms of the projected gradient using the classical redundancy formalism (yellow) and of the projected gradient using the proposed approach (blue). The projected gradient using the classical framework is lower than the one obtained with our method.

easy to see that the performance of our framework is better. The next experiment will point out that, even when DOF are available for avoidance, a better behavior of the system is obtained by considering a larger free space as done above.

The main task is composed of four visual features. The robot has to move in order to center the object in the image, to rotate it properly around the optical axis, and to bring the camera at a distance of $1.5 \mathrm{~m}$ of the target using $\mathbf{s}=\left(x_{g}, y_{g}, a_{n}, \alpha\right)$. Two DOF are thus available, that correspond mainly to motions on a sphere whose center is the target. During the servo, an object moves between the camera and the visual target, leading to an occlusion. The two available DOF are used to avoid this occlusion, as explained in Section V-C-2.

Without any avoidance law, the visual target is quickly occluded, which makes the servo to fail [Fig. 17(a)]. Using the classical redundancy formalism, the gradient is projected into a two-dimensional space. Its norm is thus reduced, and the secondary control law is not fast enough to avoid the occlusion. Mainly, the projection operator forbids the motion along the optical axis, which is controlled by one of the features of the main task (Fig. 18). This motion is available using our approach (Fig. 19). The robot velocity is thus fast enough to avoid the occlusion [Fig. 17(c)]. The decreasing speed of some visual features has been accelerated to enlarge the free space of the first task (Fig. 20). When the occlusion ends, the features decrease is no longer modified. The trajectory of the camera is given in Fig. 21. When the occlusion is not taken into account, the trajectory is a straight line. When taking the occlusion into account using the classical redundancy formalism, the additional motion 

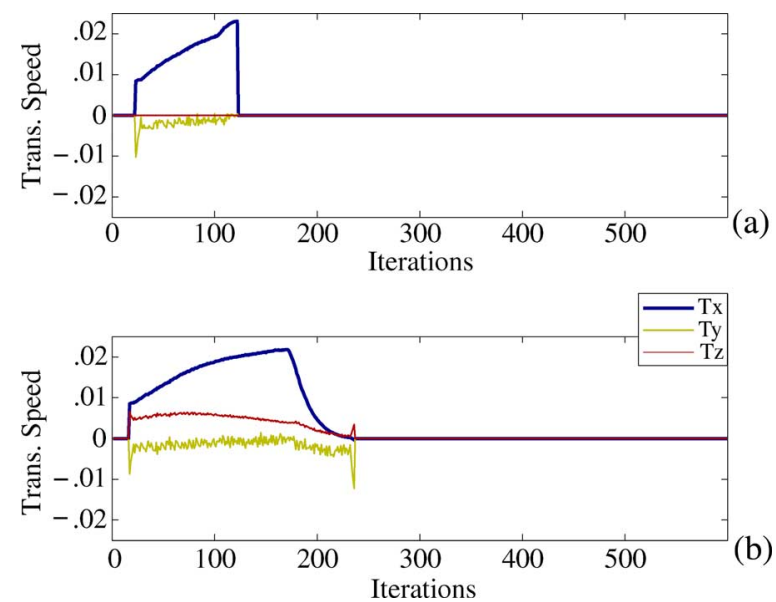

Fig. 19. Experiment C: translational velocities of the avoidance control law projected using the classical redundancy formalism (a) and projected using the proposed approach (b). The motions along the camera axis (red) are not null using the proposed control law.

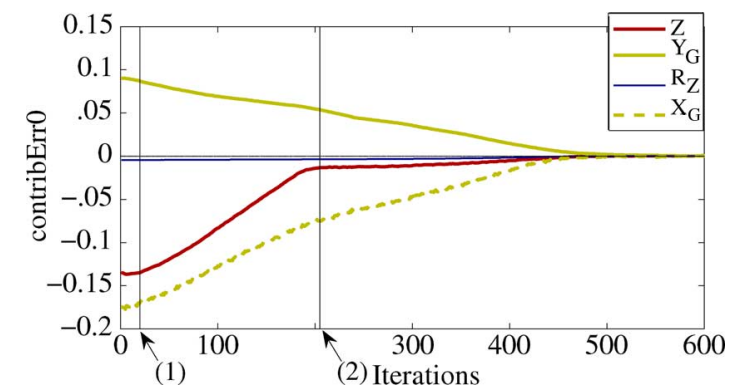

Fig. 20. Experiment C: decreasing error of the visual features. The occlusion avoidance begins at Event 1. The decrease of the feature plotted in red is accelerated. The occlusion is completely avoided after Event 2 . The decrease goes back to a normal behavior.

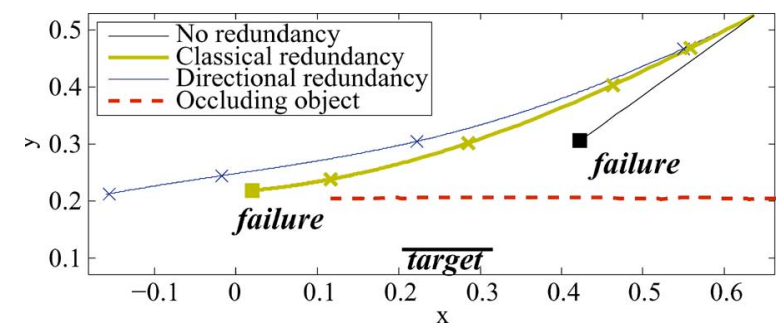

Fig. 21. Experiment C: comparison of the trajectory of the robot in the plane perpendicular to the target.

induces a translation that modifies the trajectory. When the directional redundancy is used, the robot mainly accelerates the translation toward the camera.

\section{CONCLUSION}

In this paper, we have proposed a new general method to integrate a secondary term to a first task having priority. Our framework is based on a generalization of the classical redundancy formalism. We have shown that it is possible to enlarge the number of the available DOF, and thus to improve the performance of the avoidance control law. This control scheme has been validated in simulation and on a six-DOF eye-in-hand robotic platform where the robot had to reach a specific pose with respect to a visual target, and to avoid joint limits and occlusions.
We have shown that it is possible to find DOF during the accomplishment of a full-constraining task, and to enhance the avoidance process even when enough DOF are available.

Our current works aim at realizing a reactive servo, able to perform a full constraining task and to simultaneously take into account the perturbations due to a real robotic system. The general idea is to free up as many DOF as possible to perform the avoidance of any obstacle encountered during the servo [16]. Using the method proposed in this article, additional DOF are collected at the very bottom level, directly in the control law. An avoidance can be realized even when the adequate DOF are already used by the main task. However, the number of DOF can be insufficient for example when the obstacles are numerous. We now focus on the choice of the main task, to obtain additional DOF by modifying the main task from a higher level.

\section{ACKNOWLEDGMENT}

The authors would like to thank the anonymous reviewers for their valuable comments which allow great improvements in the clarity and presentation of the paper.

\section{REFERENCES}

[1] P. Baerlocher and R. Boulic, "An inverse kinematic architecture enforcing an arbitrary number of strict priority levels," Visual Comput. vol. 6, no. 20, pp. 402-417, Aug. 2004.

[2] A. Ben-Israel and T. Greville, Generalized Inverses: Theory and Applications. New York: Wiley-Interscience, 1974.

[3] T. Chang and R. Dubey, "A weighted least-norm solution based scheme for avoiding joints limits for redundant manipulators," IEEE Trans. Robot. Automat., vol. 11, no. 2, pp. 286-292, Apr. 1995.

[4] F. Chaumette and S. Hutchinson, "Visual servo control, part i: Basic approaches," IEEE Robot. Automat. Mag., vol. 13, no. 4, pp. 82-90, Dec. 2006.

[5] F. Chaumette and E. Marchand, "A redundancy-based iterative scheme for avoiding joint limits: Application to visual servoing," IEEE Trans. Robot. Automat., vol. 17, no. 5, pp. 719-730, Oct. 2001.

[6] N. Cowan, J. Weingarten, and D. Koditschek, "Visual servoing via navigation functions," IEEE Trans. Robot. Automat., vol. 18, no. 4, pp. 521-533, Aug. 2002.

[7] B. Espiau, F. Chaumette, and P. Rives, "A new approach to visual servoing in robotics," IEEE Trans. Robot. Automat., vol. 8, no. 3, pp. 313-326, Jun. 1992.

[8] J. Feddema and O. Mitchell, "Vision-guided servoing with featurebased trajectory generation," IEEE Trans. Robot. Automat., vol. 5, no. 5, pp. 691-700, Oct. 1989.

[9] G. Hager, "Human-machine cooperative manipulation with vision-based motion constraints," in Proc. Workshop Visual Servoing, IEEE/RSJ Int. Conf. Intell. Robot. Syst. (IROS'02), Lausane, Switzerland, Oct. 2002, [CD ROM].

[10] H. Hanafusa, T. Yoshikawa, and Y. Nakamura, "Analysis and control of articulated robot with redundancy," in Proc. IFAC, 8th Triennal World Congress, Kyoto, Japan, 1981, vol. 4, pp. 1927-1932.

[11] Visual Servoing: Real Time Control of Robot Manipulators Based on Visual Sensory Feedback, K. Hashimoto, Ed. Singapore: World Scientific, 1993, vol. 7.

[12] S. Hutchinson, G. Hager, and P. Corke, "A tutorial on visual servo control," IEEE Trans. Robot. Automat., vol. 12, no. 5, pp. 651-670, Oct. 1996.

[13] O. Khatib, "Real-time obstacle avoidance for manipulators and mobile robots," Int. J. Robot. Res., vol. 5, no. 1, pp. 90-98, Spring, 1986.

[14] C. Klein and S. Kittivatcharapong, "Optimal force distribution for the legs of a walking machine with friction cone constraints," IEEE Trans. Robot. Automat., vol. 6, no. 1, pp. 73-85, Feb. 1990.

[15] A. Liégeois, "Automatic supervisory control of the configuration and behavior of multibody mechanisms," IEEE Trans. Syst., Man Cybern. vol. 7, no. 12, pp. 868-871, Dec. 1977.

[16] N. Mansard and F. Chaumette, "Task sequencing for sensor-based control," IEEE Trans. Robotics, vol. 23, no. 1, pp. 60-72, Feb. 2007.

[17] E. Marchand and G. Hager, "Dynamic sensor planning in visual servoing," in Proc. IEEE/RSJ Int. Conf. Intell. Robot. Syst. (IROS'98), Leuven, Belgium, May 1998, pp. 1988-1993. 
[18] Y. Mezouar and F. Chaumette, "Path planning for robust image-based control," IEEE Trans. Robot. Automat., vol. 18, no. 4, pp. 534-549, Aug. 2002.

[19] B. Nelson and P. Khosla, "Strategies for increasing the tracking region of an eye-in-hand system by singularity and joint limits avoidance," Int. J. Robot. Res., vol. 14, no. 3, pp. 255-269, Jun. 1995.

[20] J. Rosen, "The gradient projection method for nonlinear programmimg, part i, linear constraints," SIAM J. Appl. Math., vol. 8, no. 1, pp. 181-217, Mar. 1960.

[21] C. Samson, M. Le Borgne, and B. Espiau, Robot Control: The Task Function Approach. Oxford, U.K.: Clarendon Press, 1991.

[22] O. Schmitt, "A thermionic trigger," J. Scientific Instrum., vol. 15, pp. 24-26, 1938.

[23] O. Tahri and F. Chaumette, "Point-based and region-based image moments for visual servoing of planar objects," IEEE Trans. Robotics, vol. 21, no. 6, pp. 1116-1127, Dec. 2005.

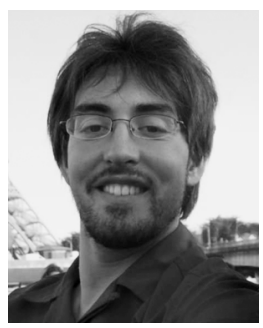

Nicolas Mansard graduated from École Nationale Supérieure d'Informatique et de Mathématiques Appliquées, Grenoble, France and received the M.S. (DEA) degree in robotics and image processing from the University Joseph Fourier, Grenoble, in 2003 and the Ph.D. degree in computer science from the University of Rennes, Rennes, France, in 2006.

He spent three years in the Lagadic research group, IRISA, INRIA-Bretagne. He then spent one year at Stanford University, Stanford, CA, and one year with JRL-Japan, AIST, Tsukuba, Japan. He is currently with LAAS/CNRS, Toulouse, France, in the GEPETTO group. His research interests are concerned with sensor-based robot animation, and more specifically the integration of reactive control schemes into real robot and humanoid applications.

Dr. Mansard received the Best Thesis Award from the French Research Group GdR-MACS, in 2006, the Best Thesis Award from the Society for Telecomunication and Computer Science (ASTI), in 2007, and the Best Thesis Award of the Région Bretagne.

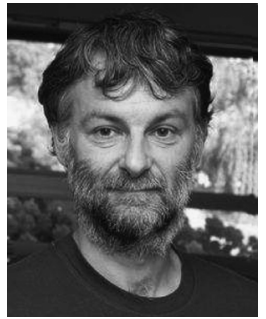

François Chaumette (M'98) received the M.S. degree from École Nationale Supérieure de Mécanique, Nantes, France, in 1987 and the Ph.D. degree in computer science from the University of Rennes, Rennes, France, in 1990.

Since 1990, he has been with IRISA/INRIA Rennes Bretagne Atlantique, where he is "Directeur de recherche" and Head of the Lagadic Group. He is the coauthor of more than 50 papers published in international journals on the topics of robotics and computer vision. His research interests include robotics and computer vision, especially visual servoing and active perception.

Dr. Chaumette received several awards including the AFCET/CNRS Prize for the Best French Thesis on Automatic Control in 1991 and the King-Sun Fu Memorial Best IEEE TRANSACTIONS ON RoBotics AND Automation Paper Award in 2002. He has been the Associate Editor of the IEEE TRANSACTIONS ON RовотіCS from 2001 to 2005 and is now in the Editorial Board of the International Journal of Robotics Research. He has served over the last five years on the program committees for the main conferences related to robotics and computer vision. 